\title{
ABELIAN AND NONDISCRETE CONVERGENCE GROUPS ON THE CIRCLE
}

\author{
A. HINKKANEN
}

\begin{abstract}
A group $G$ of homeomorphisms of the unit circle onto itself is a convergence group if every sequence of elements of $G$ contains a subsequence, say $g_{n}$, such that either (i) $g_{n} \rightarrow g$ and $g_{n}^{-1} \rightarrow g^{-1}$ uniformly on the circle where $g$ is a homeomorphism, or (ii) $g_{n} \rightarrow x_{0}$ and $g_{n}^{-1} \rightarrow y_{0}$ uniformly on compact subsets of the complements of $\left\{y_{0}\right\}$ and $\left\{x_{0}\right\}$, respectively, for some points $x_{0}$ and $y_{0}$ of the circle (possibly $x_{0}=y_{0}$ ). For example, a group of $K$-quasisymmetric maps, for a fixed $K$, is a convergence group. We show that if $G$ is an abelian or nondiscrete convergence group, then there is a homeomorphism $f$ such that $f \circ G \circ f^{-1}$ is a group of Möbius transformations.
\end{abstract}

\section{INTRODUCTION AND RESULTS}

1.1. Let $G$ be a group of homeomorphisms of the unit circle $\mathbf{T}$ onto itself. We allow the possibility that some of the homeomorphisms may be sense-reversing. The essential compactness properties of uniformly quasisymmetric or quasiconformal groups are captured by the notion of a convergence group which was recently formulated by Gehring and Martin [1, pp. 334-335]. We say that $G$ is a convergence group if every sequence of elements of $G$ contains a subsequence, say $g_{n}$, such that either

(i) $g_{n} \rightarrow g$ and $g_{n}^{-1} \rightarrow g^{-1}$ uniformly on $\mathbf{T}$, where $g$ is a homeomorphism, not necessarily an element of $G$; or

(ii) there are $x_{0}, y_{0} \in \mathbf{T}$ (possibly $x_{0}=y_{0}$ ) such that $g_{n} \rightarrow x_{0}$ and $g_{n}^{-1} \rightarrow$ $y_{0}$ uniformly on compact subsets of $\mathbf{T} \backslash\left\{y_{0}\right\}$ and $\mathbf{T} \backslash\left\{x_{0}\right\}$, respectively.

For example, if $K$ is fixed, a group of $K$-quasisymmetric functions is a convergence group.

A Möbius group is a group of Möbius transformations of $\mathbf{T}$, or of the real axis $\mathbf{R}$, or of the extended real axis $\overline{\mathbf{R}}=\mathbf{R} \cup\{\infty\}$ onto itself. We allow a Möbius transformation to be sense-reversing. We remark that a homeomorphism $f$ of $\overline{\mathbf{R}}$ is sense-preserving if and only if $f$ is increasing on $\mathbf{R}$ with $f(\infty)=\infty$, or if $f$ is increasing on $(-\infty, a)$ and on $(a, \infty)$ where $a=f^{-1}(\infty)$ is finite.

A group $G$ is nondiscrete if $G$ contains a sequence $g_{n}$ of distinct elements such that $g_{n} \rightarrow$ Id uniformly on $\mathbf{T}$ or on $\overline{\mathbf{R}}$. Here and later, Id denotes the

Received by the editors February 3, 1988.

1980 Mathematics Subject Classification (1985 Revision). Primary 30C60.

Research partially supported by the U.S. National Science Foundation. 
identity mapping of any set. Otherwise, a group is discrete, so that the limit of a sequence of distinct elements of $G$ cannot be a homeomorphism, and only the alternative (ii) in the definition of a convergence group can then occur for such a sequence.

If $Z=\left(z_{1}, z_{2}, z_{3}, z_{4}\right)$ is a quadruple of distinct points of $\mathbf{T}$ following each other in the positive or negative direction, we define the cross ratio $C(Z)$ of $Z$ by

$$
C(Z)=\frac{z_{4}-z_{1}}{z_{4}-z_{2}} \frac{z_{3}-z_{2}}{z_{3}-z_{1}}
$$

so that $0<C(Z)<1$. We say that the homeomorphism $f$ of $\mathbf{T}$ is $K$ quasisymmetric $(K$-qs $)$ if

$$
(2 K)^{-1} \leq C(f Z) \leq 1-(2 K)^{-1}
$$

whenever $C(Z)=\frac{1}{2}$. Here $K \geq 1$ and, of course, $f Z=\left(f\left(z_{1}\right), f\left(z_{2}\right), f\left(z_{3}\right)\right.$, $\left.f\left(z_{4}\right)\right)$. A group $G$ is $K-q s$ if all the elements of $G$ are $K$-qs. We say that $G$ is a uniformly quasisymmetric group if $G$ is a $K$-qs group for some $K$.

1.2. The main structural problem concerning convergence groups on $\mathbf{T}$ is whether or not each such group $G$ is topologically conjugate to a Möbius group, that is, if there is a homeomorphism $f$ such that $f \circ G \circ f^{-1}$ is a Möbius group. In [3], we proved that this is the case if $G$ is a nondiscrete or abelian $K$-quasisymmetric group, or if $G$ is a $K$-qs group all of whose elements have a common fixed point. In [5], Tukia has shown that a large class of discrete convergence groups are topological conjugates of Möbius groups. We remark that in [4, Theorem 4.4], Martin and Tukia showed that any discrete convergence group on the closed unit disk is topologically conjugate to a Fuchsian group.

In this paper we prove the following result.

Theorem 1. Let $G$ be a convergence group on the unit circle. There is a homeomorphism $f$ such that $f \circ G \circ f^{-1}$ is a group of Möbius transformations provided that one of the following holds:

(i) all functions in $G$ have a common fixed point;

(ii) $G$ is abelian (in particular if $G$ is cyclic);

(iii) $G$ is a finite group;

(iv) $G$ is nondiscrete; or

(v) $G$ is uncountable (and hence nondiscrete).

Hence Theorem 1 is a generalization of the results in [2] and [3] for convergence groups. Theorem 1 overlaps to some extent with Tukia's results in [5] as the latter apply to all finite groups and all abelian discrete groups.

1.3. It is simpler to solve the conjugacy problem for convergence groups than for $K$-qs groups in the sense that for convergence groups, the function $f$ in Theorem 1 is only required to be a homeomorphism, while if $G$ is a $K$-qs group, one normally wants to find a $K_{1}$-qs function $f$, where $K_{1}$ depends on $K$ only, such that $f \circ G \circ f^{-1}$ is a Möbius group. 
However, many proofs of auxiliary results in [3] and in the earlier paper [2], on whose results we essentially relied many times in [3], depended on compactness properties of the conjugating quasisymmetric functions, and these are no longer available for convergence groups. In [2] and [3], we often considered a sequence $G_{n}$ of $K$-qs groups and a corresponding sequence $f_{n}$ of suitably normalized $K_{1}$-qs functions such that $f_{n} \circ G_{n} \circ f_{n}^{-1}$ is a Möbius group for all $n$. We were able to deduce that a subsequence of $f_{n}$ tends to $K_{1}$-qs map $f$, which then conjugated some $K$-qs group to a Möbius group.

Suppose now only that the $G_{n}$ are convergence groups and that the $f_{n}$ are homeomorphisms. Then, even though convergence groups have certain compactness properties, there is no obvious guarantee that any subsequence of the $f_{n}$ would converge to a homeomorphism even if all the $f_{n}$ fix three given points. It is not clear to me how such guarantees could arise from the fact that the $f_{n}$ conjugate to Möbius groups, the groups $G_{n}$ that are, in some way, related to each other. Therefore, some of the methods that we shall develop are quite different from those used in [2] and [3].

There are, however, arguments, lemmas, and entire sections of [2] and [3] that can be used in the proof of Theorem 1 if they apply, essentially word by word, to convergence groups. If such arguments rely on lemmas for uniformly quasisymmetric groups, we can still appeal to them, provided that we have proved similar lemmas for convergence groups. We shall feel free to quote [2] and [3] in this way, whenever possible.

1.4. Finally, for the sake of completeness, we record the following simple result.

Theorem 2. Let $G$ be a finite $K$-quasisymmetric group on the unit circle. Then there is a $K_{1}$-quasisymmetric function $f$, where $K_{1}$ depends on $K$ only, such that

$$
f \circ G \circ f^{-1}=\left\{z \mapsto e^{2 \pi i n / N} z: 1 \leq n \leq N\right\}=G_{1},
$$

say, or

$$
f \circ G \circ f^{-1}=G_{1} \cup\left\{z \mapsto e^{2 \pi i n / N} \bar{z}: 1 \leq n \leq N\right\} .
$$

If $G$ is a finite convergence group on the circle, there is a homeomorphism $f$ such that $f \circ G \circ f^{-1}$ is given by (1.1) or (1.2).

Theorem 2 is relatively easy to prove for finite convergence groups as they are either cyclic or contain a cyclic subgroup of index two. For $K$-quasisymmetric groups, the main problem is to construct a $K_{1}$-quasisymmetric homeomorphism $f$ such that (1.1) or (1.2) holds, where $K_{1}$ depends on $K$ only (and not, for example, also on the order of the group $G$ ). It is not hard to define $f$, but the proof that $f$ is $K_{1}$-qs is tedious.

Theorem 1 will be proved in many stages, in $\S \S 2-8$. We shall prove Theorem 2 in the course of this work in $\S 7$. In $\S 2$ we prove some preliminary lemmas 
and deal with cyclic groups. In $\S 3$ we show that we may assume the group $G$ to be closed in a certain sense. Then we shall outline the proof of Theorem 1 in $\S 4$, and go through the details in $\S \S 5-8$.

I wish to thank the referee for his suggestions concerning the exposition.

\section{CYClic GROUPS}

2.1. Notation. We denote the identity mapping of any set by Id. If $g$ is a homeomorphism of a set onto itself, we write $g^{0}=$ Id and define $g^{n}=g \circ g^{n-1}$ and $g^{-n}=\left(g^{n}\right)^{-1}$ for $n \geq 1$. If $s$ is real and $b \neq 0$, we set

$$
\begin{aligned}
T_{s}(x) & =x+s \text { for } x \in \mathbf{R}, \\
M_{b}(x) & =b x \text { for } x \in \mathbf{R}, \\
R_{s}(z) & =[\exp (2 \pi i s)] z \text { for }|z|=1 .
\end{aligned}
$$

We write $\mathscr{T}$ for the group $\left\{T_{s}: s \in \mathbf{R}\right\}$ of all translations and $\mathscr{M}$ for the group $\left\{M_{b}: b>0\right\}$ of all increasing dilations of $\mathbf{R}$. The group $\left\{R_{\alpha}: \alpha \in \mathbf{R}\right\}=$ $\left\{R_{c}: 0 \leq \alpha<1\right\}$ of all rotations of the unit circle $\mathbf{T}$ is denoted by $\mathscr{R}$.

We write $e(x)=\exp (2 \pi i x)$. If $\theta_{1}<\theta_{2}<\theta_{1}+2 \pi$ and $a_{i}=e\left(\theta_{i}\right)$ for $i=1,2$, we write

$$
\left(a_{1}, a_{2}\right)=\left\{e(\theta): \theta_{1}<\theta<\theta_{2}\right\} .
$$

We denote the corresponding closed and half-closed arcs by $\left[a_{1}, a_{2}\right],\left[a_{1}, a_{2}\right)$, and $\left(a_{1}, a_{2}\right]$. If $I$ is an arc of $\mathbf{T}$ or a subinterval of $\mathbf{R}$, we denote the length of $I$ by $|I|$.

Let $h$ be a Möbius transformation of $\mathbf{T}$ onto $\overline{\mathbf{R}}$. Then $G$ is a convergence group on $\mathbf{T}$ if and only if $h \circ G \circ h^{-1}=G_{1}$ is a convergence group on $\overline{\mathbf{R}}$. If all elements of $G$ fix $z_{0} \in \mathbf{T}$, we may choose $h$ so that $h\left(z_{0}\right)=\infty$, and then all elements of $G_{1}$ fix $\infty$. Most properties that we shall be interested in, such as being conjugate to a Möbius group, are preserved under conjugation, so that such properties are the same for $G$ and $G_{1}$. For this reason, we shall feel free to consider a group on $\overline{\mathbf{R}}$ instead of $\mathbf{T}$ whenever this is convenient and without further justification. Also we shall assume, whenever appropriate, that some or all elements of $G$ fix the point at infinity.

Whether we work on $\mathbf{T}$ or on $\overline{\mathbf{R}}$, we may replace $G$ by $h_{1} \circ G \circ h_{1}^{-1}$ where $h_{1}$ is a Möbius transformation taking $\mathbf{T}$ or $\overline{\mathbf{R}}$ onto itself. We may choose $h_{1}$ to take given three distinct points onto some other given three distinct points which, on $\overline{\mathbf{R}}$, might be the point at infinity together with two finite points. We shall also feel free to make use of this observation.

2.2. Lemmas on fixed points. We first develop some basic results about the elements of a convergence group. Lemmas 1 and 2 below are analogous to Lemmas 1 and 2 in [2] for quasisymmetric groups. Tukia [5] also observed that these two results are valid for convergence groups. We give the proofs for the sake of completeness. 
Lemma 1. If $g$ generates a convergence group and $g \neq \mathrm{Id}$, then $g$ has at most two fixed points on the circle.

Proof. A sense-reversing function has exactly two fixed points. Hence we may assume that $g$ is sense-preserving, $g \neq \mathbf{I d}$, and $g$ has at least three fixed points. We may consider the situation on $\overline{\mathbf{R}}$ and assume further that $g$ fixes 0,1 , and $\infty$ but that $g$ fixes no point on $(0,1)$. Replacing $g$ by $g^{-1}$, if necessary, we may assume that $g(x)>x$ for $0<x<1$. Hence $g^{n}(x) \rightarrow 1$ as $n \rightarrow \infty$ for all $x \in(0,1]$.

Since $g$ generates a convergence group, it follows from the above and from the definition of a convergence group that $g^{n_{j}}(x) \rightarrow 1$ locally uniformly on $\overline{\mathbf{R}} \backslash\left\{y_{0}\right\}$, for some $y_{0} \in \overline{\mathbf{R}}$ and some subsequence $g^{n_{j}}$. This is impossible since each $g^{n}$ fixes 0 and $\infty$, and Lemma 1 is proved.

Lemma 2. Suppose that $g$ and $h$ are elements of the convergence group $G$ and that $z_{1}, z_{2}$, and $z_{3}$ are distinct points of the circle. If $g\left(z_{i}\right)=h\left(z_{i}\right)$ for $1 \leq i \leq 3$, then $g=h$.

If $\bar{g}$ and $h$ are defined on $\overline{\mathbf{R}}$, if $g(\infty)=h(\infty)$ and $g\left(x_{0}\right)=h\left(x_{0}\right)$ where $x_{0} \in \mathbf{R}$, and if $g(x)<h(x)$ for $x<x_{0}$, then $g(x)>h(x)$ for $x>x_{0}$.

Proof. The function $h_{1}=g^{-1} \circ h \in G$, and $h_{1}\left(z_{i}\right)=z_{i}$ for $1 \leq i \leq 3$. Hence $h_{1}=$ Id by Lemma 1 , and so $g=h$.

If the second statement is not true, we must have $g(x)<\bar{h}(x)$ for $x \neq x_{0}$, so that $h_{1}(\infty)=\infty, h_{1}\left(x_{0}\right)=x_{0}$, and $h_{1}(x)>x$ for $x \neq x_{0}$. But then $h_{1}^{n}(x) \rightarrow \infty$ as $n \rightarrow \infty$ for all $x>x_{0}$ while $h_{1}^{n}(x) \rightarrow x_{0}$ as $n \rightarrow \infty$ for all $x<x_{0}$. Since $h_{1}$ generates a convergence group, this leads to a contradiction, in view of the definition of a convergence group. Lemma 2 is proved.

Lemma 3. Suppose that $g$ generates a convergence group and that $g$ has no fixed points on the circle. Then, if $N$ is a nonzero integer and $g^{N}$ has a fixed point, we have $g^{N}=\mathrm{Id}$.

Hence either $g$ is of finite order, or $g^{n}$ has no fixed points for any $n \neq 0$.

Lemma 3 is proved in the same way as [3, Lemma 4] by using the above Lemmas 1 and 2 . Hence we omit the proof.

2.3. Proof of Theorem 1 for cyclic groups. For easier reference, we state this as a lemma.

Lemma 4. A cyclic convergence group is topologically conjugate to a Möbius group.

Proof. Let the group be $G$, generated by $g$. We may assume that $g \neq$ Id. We need only find a homeomorphism $f$ such that $f \circ g \circ f^{-1}$ is a Möbius transformation. In view of Lemmas 1 and 3, the following six cases can occur:

(i) $g^{n}$ has no fixed points for any $n \neq 0$;

(ii) $g$ has no fixed points but $g^{N}=$ Id for some $N \geq 2$; 
(iii) $g$ has exactly one fixed point;

(iv) $g$ is sense-preserving with two fixed points;

(v) $g$ is sense-reversing with $g^{2}=\mathrm{Id}$; or

(vi) $g$ is sense-reversing with $g^{2} \neq \mathrm{Id}$.

Note that for topological reasons, a sense-reversing map of $\mathbf{T}$ or of $\overline{\mathbf{R}}$ onto itself has exactly two fixed points.

Cases (ii) to (vi) are covered by [5, Theorem 2A], but we prefer to give a more detailed proof. Therefore we shall now go through the six cases one by one.

In case (i), we deduce that $g$ is a so-called minimal homeomorphism and that therefore there is a homeomorphism $f$ such that $f \circ g \circ f^{-1}=R_{\alpha}$ for an irrational number $\alpha \in(0,1)$, the rotation number of $g$. Furthermore, $f$ is uniquely determined up to a rotation; that is, $f$ could only be replaced by $R_{\beta} \circ f$ where $\beta$ is real, and any real $\beta$ would do. All of this was proved in [3, pp. 60-62] under the assumption that $g$ generates a uniformly quasisymmetric group. However, as mentioned in [3, p. 62], Gaven Martin has observed that the same proof goes through when $g$ generates a convergence group, and the modifications were explained in [3, p. 62].

In case (ii), we choose $N \geq 2$ with $g^{N}=$ Id to be as small as possible, so that $N$ is the order of $g$. As in [3, pp. 29-30], we note that by replacing $g$ by $g^{l}$, if necessary, where $1 \leq l \leq N-1$ and $g^{l}$ generates the same group as $g$, we may assume that for all $z \in \mathbf{T}$, the points $z, g(z), g^{2}(z), \ldots, g^{N-1}(z)$ follow each other in this order as we trace $\mathbf{T}$ in the positive direction. To find a homeomorphism $f$ such that $f^{-1} \circ g \circ f=R_{1 / N}$, that is,

$$
f(c z)=g(f(z)) \text { for } z \in \mathbf{T},
$$

where $c=e(1 / N)$, we define $f(e(\theta))=e(\psi(\theta))$ for $0 \leq \theta \leq 1 / N$, where $\psi$ is linear on $[0,1 / N]$ with $\psi(0)=0$ and

$$
0<\psi(1 / N)<1, \quad e(\psi(1 / N))=g(1) .
$$

Then (2.2) extends $f$ to a homeomorphism of $\mathbf{T}$ onto itself such that $f^{-1} \circ G \circ f$ is a Möbius group.

In case (iii), we may assume that $g$ is defined on $\overline{\mathbf{R}}$ with $g(\infty)=\infty$. Replacing $g$ by $g^{-1}$, if necessary, we may assume that $g(x)>x$ for all real $x$. Given any positive $r$, we find a homeomorphism $f$ of $\overline{\mathbf{R}}$ onto itself with $f(\infty)=\infty$ and $f^{-1} \circ g \circ f=T_{r}$ as follows. We require that

$$
f(x+r)=g(f(x)) \text { for all } x \in \mathbf{R} \text {. }
$$

We set $f(0)=0, f(r)=g(0)>0$, and take $f$ to be linear on $[0, r]$. Indeed, we could take $f \mid[0, r]$ to be any increasing homeomorphism of $[0, r]$ onto $[0, g(0)]$, and we could choose $s, t \in \mathbf{R}$ and replace $[0, r],[0, g(0)]$ by $[s, s+r]$ and $[t, g(t)]$, respectively. Now (2.3) extends $f$ to an increasing homeomorphism of $\overline{\mathbf{R}}$ onto itself satisfying (2.3). Note that for all real $x$ and 
all integers $n$, we have $g^{n}(x)<g^{n+1}(x)$ and further that $g^{n}(x) \rightarrow \infty$ as $n \rightarrow \infty$ and that $g^{n}(x) \rightarrow-\infty$ as $n \rightarrow-\infty$ since $g$ has no finite fixed points. This proves Lemma 4 in case (iii).

In case (iv), we may assume that $g$ is defined on $\overline{\mathbf{R}}$, that $g$ fixes 0 and $\infty$, and that $g(x)>x$ for $x>0$. Then $g(x)<x$ for $x<0$ by Lemma 2. We choose a number $b>1$ and find a homeomorphism $f$ fixing 0 and $\infty$ such that $f^{-1} \circ g \circ f=M_{b}$, that is,

$$
f(b x)=g(f(x)) \text { for all real } x .
$$

For example, we may take $f$ to be linear on $[-b,-1]$ and on $[1, b]$ with $f(1)=1=-f(-1)$ and $f(b)=g(1)>1, f(-b)=g(-1)<-1$. Then (2.4) extends $f$ to $\overline{\mathbf{R}}$, and $f^{-1} \circ G \circ f$ is a Möbius group.

In case $(\mathbf{v})$, we may assume that $g$ is defined and strictly decreasing on $\mathbf{R}$ and that $g$ fixes 0 and $\infty$. We set $f(x)=x$ for $0 \leq x \leq \infty$ and $f(x)=-g(x)$ for $-\infty<x<0$. Then $\left(f \circ g \circ f^{-1}\right)(x)=-x$ for all real $x$ since $g^{2}=$ Id .

In case (vi), we may assume that $g$ is decreasing on $\mathbf{R}$ with fixed points 0 and $\infty$. Then $g^{2}$ is sense-preserving and hence as in case (iv) above. We may therefore perform a preliminary conjugation and assume that $g^{2}(x)=4 x$ for $x \in \mathbf{R}$. We define $f(x)=x$ for $0 \leq x \leq \infty$ and $f(x)=-g(x) / 2$ for $-\infty<x<0$. Then $\left(f \circ g \circ f^{-1}\right)(x)=-2 x$ for all real $x$. This completes the proof of Lemma 4.

\section{THE CLOSURE OF A CONVERGENCE GROUP}

We may consider a convergence group $G$ on $\mathbf{T}$ to be a subgroup of the topological group of all homeomorphisms of $\mathbf{T}$ onto itself in the compactopen topology, that is, in the topology of uniform convergence on $\mathbf{T}$. So the homeomorphism $h$ is a limit point of $G$ if there is a sequence $g_{n}$ of distinct elements of $G$ such that $g_{n} \rightarrow h$ uniformly on $\mathbf{T}$. The closure $\bar{G}$ of $G$ is the union of $G$ and the set of all limit points of $G$.

When proving Theorem 1 for noncyclic groups, it will often be convenient for us to assume that $G$ is closed. This is made possible by the following lemma.

Lemma 5. If $G$ is a convergence group on $\mathbf{T}$, then so is $\bar{G}$.

If $f$ is a homeomorphism such that $f \circ \bar{G} \circ f^{-1}$ is a Möbius group, then $f \circ G \circ f^{-1}$ is also a Möbius group. If $G$ is nondiscrete, abelian, or uncountable, then so is $\bar{G}$. If all the functions in $G$ have a common fixed point $z_{0}$, then also each element of $\bar{G}$ fixes $z_{0}$. Hence we may always assume in the sequel that $G$ is closed.

Proof. To prove Lemma 5, let $h_{n}$ be a sequence of elements of $\bar{G}$, and for each $n$, let $g_{m n}$ be a sequence in $G$ such that $g_{m n} \rightarrow h_{n}$ uniformly on $\mathbf{T}$ as $m \rightarrow \infty$. We write

$$
\|f-g\|_{E}=\sup \{|f(z)-g(z)|: z \in E\}
$$


when $E \subset \mathbf{T}$, and $\|f-g\|=\|f-g\|_{\mathbf{T}}$. By passing to a subsequence, for each $n$, and using (i) of the definition of a convergence group, we may assume that $\left\|g_{m n}-h_{n}\right\|<2^{-m}$ and $\left\|g_{m n}^{-1}-h_{n}^{-1}\right\|<2^{-m}$ for all $m$ and $n$.

We write $f_{n}=g_{n n} \in G$. We may choose a subsequence $f_{n(k)}$, where $n(k) \geq k$, that satisfies (i) or (ii) of the definition of a convergence group. If (i) holds, then $f_{n(k)} \rightarrow f$ and $f_{n(k)}^{-1} \rightarrow f^{-1}$ uniformly on $\mathbf{T}$, where $f$ is a homeomorphism. Thus also $h_{n(k)} \rightarrow f$ and $h_{n(k)}^{-1} \rightarrow f^{-1}$ since, for example,

$$
\begin{aligned}
\left\|h_{n(k)}-f\right\| & \leq\left\|h_{n(k)}-f_{n(k)}\right\|+\left\|f_{n(k)}-f\right\| \\
& \leq 2^{-n(k)}+\left\|f_{n(k)}-f\right\| \rightarrow 0
\end{aligned}
$$

as $k \rightarrow \infty$.

If (ii) holds, then $f_{n(k)} \rightarrow x_{0}$ and $f_{n(k)}^{-1} \rightarrow y_{0}$ uniformly on compact subsets of $\mathbf{T} \backslash\left\{y_{0}\right\}$ and $\mathbf{T} \backslash\left\{x_{0}\right\}$, respectively. Let $E$ be a compact subset of $\mathbf{T} \backslash\left\{y_{0}\right\}$, and note that $\|f-g\|_{E} \leq\|f-g\|$ for any $E \subset \mathbf{T}$. Then

$$
\begin{aligned}
\left\|h_{n(k)}-x_{0}\right\|_{E} & \leq\left\|h_{n(k)}-f_{n(k)}\right\|_{E}+\left\|f_{n(k)}-x_{0}\right\|_{E} \\
& \leq 2^{-n(k)}+\left\|f_{n(k)}-x_{0}\right\|_{E} \rightarrow 0
\end{aligned}
$$

as $k \rightarrow \infty$. Hence $h_{n(k)} \rightarrow x_{0}$ uniformly on $E$, and similarly $h_{n(k)}^{-1} \rightarrow y_{0}$ uniformly on compact subsets of $\mathbf{T} \backslash\left\{x_{0}\right\}$. This proves Lemma 5 .

\section{OUtLINE OF THE PROOF OF THEOREM 1}

4.1. Case (i). To prove (i) of Theorem 1, we proceed roughly as follows. We may assume that $G$ is closed, that $G$ is defined on $\overline{\mathbf{R}}$, and that all elements of $G$ fix the point at infinity. Let $G_{T}$ be the subset of $G$ consisting of Id together with those elements of $G$ that have no finite fixed points. In $\S \S 5$ and 6 we shall show that $G_{T}$ is a subgroup of $G$ and that $G_{T}$ can be conjugated to a group of translations.

Suppose for a while that $G$ contains no sense-reversing (that is, decreasing) functions. Suppose further that $G \neq G_{T} \neq\{$ Id $\}$. We shall show in $\S 5$ that then $G_{T}$ is not cyclic since $G$ is not cyclic, and that the function conjugating $G_{T}$ to a group of translations (indeed, to all of $\mathscr{T}$ ) also conjugates $G$ to a Möbius group.

We shall show in Lemma 9 that if $g, h \in G$ fix different finite points then $g \circ h \circ g^{-1} \circ h^{-1}$ is in $G_{T} \backslash\{\mathrm{Id}\}$. Hence, if $G_{T}=\{\mathrm{Id}\}$, all functions in $G$ have the same finite fixed point, say the origin. We shall show in $\S 6$ that then $G$ can be conjugated to a subgroup of all dilations. In a sense, this is very similar to the problem of conjugating $G_{T}$ to a group of translations.

If $G$ contains decreasing functions, then, by the above, we may assume that the subgroup of $G$ consisting of increasing functions is a Möbius group. As we shall explain at the end of $\S 6$, then $G$ can be conjugated to a Möbius group, essentially following the arguments in $[3, \S 3]$. 
4.2. Case (iv). Theorem 1 will be proved separately for finite groups (case (iii)) in $\S 7$, while after proving Theorem 1 for nondiscrete groups (case (iv)), it is easy to extend it to abelian groups and uncountable groups by following the arguments given in [3]. We now outline the proof of Theorem 1 for nondiscrete closed convergence groups $G$. There is a sequence $g_{n}$ of distinct elements of $G$ tending to Id. We may take the $g_{n}$ to be sense-preserving, and then by Lemma 4 each $g_{n}$ is topologically conjugate to a Möbius transformation, which may be hyperbolic, parabolic, or elliptic. We use the same terminology of the functions $g_{n}$ themselves.

By passing to a subsequence, we may assume that the $g_{n}$ are all of the same type.

Since $G$ contains all the iterates of each $g_{n}$ and since $G$ is closed, it turns out that $G$ contains a subgroup topologically conjugate to one of $\mathscr{T}, \mathscr{M}$, and $\mathscr{R}$. This will be proved in Lemma 14 in $\S 8$ below. By performing a preliminary conjugation, we may thus assume that $G$ contains $\mathscr{T}, \mathscr{M}$, or $\mathscr{R}$. In $[3, \S 10]$, it was shown that if $G$ is a uniformly quasisymmetric group containing $\mathscr{T}$ or $\mathscr{R}$, then $G$ is a Möbius group, and that if $G$ is such a group containing $\mathscr{M}$ (and thus defined on $\overline{\mathbf{R}}$ ) then $G$ can be conjugated to a Möbius group by a function $f$ of the form

$$
f(x)=A x^{r} \quad \text { for } 0 \leq x \leq \infty
$$

and

$$
f(x)=-B|x|^{r} \text { for }-\infty<x<0
$$

where $A, B$, and $r$ are positive numbers.

In the arguments in $[3, \S 10]$, we made no essential use of the assumption that $G$ is a $K$-qs group. The same proof applies, word by word, to convergence groups. Thus we shall not repeat it in this paper. Our main problem, then, is to show that $G$ indeed contains a group conjugate to one of $\mathscr{T}, \mathscr{M}$, and $\mathscr{R}$. When all the functions $g_{n}$ are parabolic or hyperbolic, this is not too hard to do. However, much more care needs to be exercised when all the $g_{n}$ are elliptic, and the bulk of $\S 8$ is taken up by this case. This concludes our outline of the proof of Theorem 1 in case (iv).

\section{AUXILIARY RESULTS FOR THE TRANSLATION SUBGROUP}

5.1. Suppose that the convergence group $G$ is defined on $\overline{\mathbf{R}}$ and that all functions in $G$ fix infinity. Recall that $G_{T}$ consists of Id and those elements of $G$ that have no finite fixed points. In this section we shall establish a few auxiliary results related to $G_{T}$.

Lemma 6. If $g, h \in G_{T}$ and $g \neq h$, then $g(x) \neq h(x)$ for all real $x$. Hence $g^{-1} \circ h$ belongs to $G_{T}$, and $G_{T}$ is a subgroup of $G$. Also $G_{T}$ is a convergence group.

Lemma 7. If $G$ is not cyclic and if $G \neq G_{T} \neq\{\mathrm{Id}\}$, then $G_{T}$ is not cyclic. If $G$ is also closed, then $G_{T}$ is closed. 
The proof of Lemma 7 is essentially the same as that of [2, Lemma 8, p. 334]. Hence we omit it.

Lemma 8. Suppose that $G_{1}=\left\{T_{s}: s \in S\right\}$ is a subgroup of $G$ where $S$ is a dense subset of $\mathbf{R}$. Then every element $g$ of $G$ is linear, of the form $g(x)=a x+b$ where $a$ and $b$ are real and $a \neq 0$. This applies also if $G$ contains sensereversing functions.

The proof of Lemma 8 is the same as that of [2, Lemma 9, p. 334], so we omit it.

Lemma 9. If $g$ and $h$ are increasing functions that belong to $G \backslash\{\mathrm{Id}\}$ and fix different finite points, then $H=g \circ h \circ g^{-1} \circ h^{-1}$ has no finite fixed points. Hence $H \in G_{T} \backslash\{\mathrm{Id}\}$.

Lemmas 6 and 9 correspond to Lemmas 7 and 6 in [2], which were proved rather quickly by means of the corollary at the end of $\S 3$ in [2, p. 331]. This corollary is not always true for convergence groups, and so we have to resort to quite different arguments. Unfortunately, the simplest proof that $I$ have been able to find for Lemma 6 is rather involved. For example, I do not even know of a short proof for the fact that the functions $x+1$ and $x+e^{x}$ do not generate a convergence group. That group certainly cannot be conjugated to a Möbius group, since $x+1$ and $x+e^{x}$ have no finite fixed points and would have to correspond to translations, while their graphs intersect at $x=0$, which cannot happen to two distinct translations. The problem seems to lie in the fact that $x+e^{x}$ grows very rapidly as $x \rightarrow \infty$, indeed faster than any quasisymmetric function.

Thus the proof of Lemma 6 is one of the main points where we introduce new methods that cannot be found already in [2] and [3]. The proof of Lemma 9 is somewhat simpler than that of Lemma 6.

5.2. Proof of Lemma 6. The idea of the proof is as follows. To get a contradiction with Lemma 2, we find $g_{1}, g_{2} \in G_{T}$ such that $g_{1} \neq g_{2}$ but the graphs of $g_{1}$ and $g_{2}$ intersect at least twice (at finite points). To do this, we need to find $g_{1}, g_{3} \in G_{T}$ whose growth, as $x \rightarrow \infty$, is so different that for any $n \geq 1$, we have $g_{1}(x)>g_{3}^{n}(x)$ for all large $x$. On the other hand, since $g_{3}^{n}(0) \rightarrow \infty$ as $n \rightarrow \infty$, the graphs of $g_{1}$ and $g_{3}^{n}$ intersect if $n$ is large enough. By Lemma 2, we then have $g_{1}(x)<g_{3}^{n}(x)$ for $x<x_{n}$, say. Further, $g_{1}$ and $g_{3}$ will be constructed so that $g_{1}(x)-x$ and $g_{3}^{n}(x)-x$ tend to zero as $x \rightarrow-\infty$, for all $n \geq 1$. Then $\left(g_{1} \circ g_{3}^{-n}\right)(x)-x$ is negative for $x<g_{3}^{n}\left(x_{n}\right)$ and tends to zero as $x \rightarrow-\infty$. Sublemma 1 below says that such a situation cannot occur, which gives the desired contradiction. It turns out that the choice $g_{3}=T_{-1} \circ g_{1} \circ T_{1} \circ g_{1}^{-1}$ yields a function growing more slowly than $g_{1}$.

5.2.1. To prove Lemma 6, suppose that $g$ and $h$ are distinct functions in $G_{T}$. If $h(x) \leq x<g(x)$ for all $x$, there is nothing to prove. By passing to 
inverse functions, if necessary, we may assume that $g(x)>x$ and $h(x)>x$ for all $x$.

We may assume that $G$ is closed, by Lemma 5. Recall that $T_{s}(x)=x+s$ for $x, s \in \mathbf{R}$. Suppose that $g\left(x_{0}\right)=h\left(x_{0}\right)$ for some finite $x_{0}$. By Lemma 4, and by interchanging $g$ and $h$, if necessary, we may perform a preliminary conjugation and assume that $h=T_{1}$ and that $x<g(x)<x+1$ for $x<x_{0}$. Then $g(x)>x+1$ for $x>x_{0}$, by Lemma 2 .

We proceed by proving a series of claims formulated as sublemmas. For $\varphi \in G$, we define $F(\varphi)=T_{-1} \circ \varphi \circ T_{1} \circ \varphi^{-1} \in G$. We denote by $\mathscr{F}$ the set of functions $\varphi \in G_{T}$ such that graphs of $\varphi$ and $T_{1}$ intersect at some finite point, say $x_{1}$, and such that $x<\varphi(x)<x+1$ for $x<x_{1}$. Then $\varphi(x)>x+1$ for $x>x_{1}$. Eventually, we need to prove that $\mathscr{F}$ is empty.

5.2.2. Sublemma 1. If $T_{1} \in G$ then there is no $\omega \in G$ such that $\omega$ has a finite fixed point $x_{0}$, such that $\omega(x)>x$ for $x>x_{0}$, and such that

$$
\lim _{x \rightarrow-\infty}(\omega(x)-x)=0 \text {. }
$$

Proof. One can verify that if $(5.1)$ holds, then $\omega^{q}(x)-x \rightarrow 0$ as $x \rightarrow-\infty$, for each $q \geq 1$. Hence $\omega^{q}(x)>x-1$ for $x<x_{1}(q)$, say. Set $x_{2}=x_{0}-1$. Then $\omega^{q}\left(x_{2}\right) \rightarrow-\infty$ as $q \rightarrow \infty$ so that $\omega^{q}\left(x_{2}\right)<x_{2}-1$ for some $q$. Since $\omega^{q}(x)>x$ for $x>x_{0}$, it follows that the graphs of the distinct elements $\omega^{q}$ and $T_{-1}$ of $G$ have two finite points of intersection, which is against Lemma 2. This proves Sublemma 1.

Sublemma 2. If $\varphi \in \mathscr{F}$ then $F(\varphi) \neq$ Id. Further, we cannot have $\varphi(x+1)<$ $\varphi(x)+1$ for all $x$.

Proof. If $F(\varphi)=$ Id then $\varphi$ commutes with $T_{1}$. There is $x_{1}$ such that $\varphi\left(x_{1}\right)=$ $x_{1}+1$, and it follows that $\varphi\left(x_{1}+n\right)=T_{1}\left(x_{1}+n\right)$ for all integers $n$. By Lemma 2 , we have $\varphi=T_{1}$, which is against our assumption. Hence $F(\varphi) \neq \mathrm{Id}$.

If $\varphi(x+1)<\varphi(x)+1$ for all $x$, let $x_{1}$ be the point with $\varphi\left(x_{1}\right)=x_{1}+1$. Then $\varphi\left(x_{1}-1\right)<\left(x_{1}-1\right)+1=x_{1}$ and hence $\varphi\left(x_{1}\right)<\varphi\left(x_{1}-1\right)+1<x_{1}+1$, which is a contradiction. This proves Sublemma 2.

By Lemma 2, it now follows for $\varphi \in \mathscr{F}$ that either $F(\varphi)(x)>x$, that is,

$$
\varphi(x+1)>\varphi(x)+1 \text { for all } x,
$$

or $F(\varphi)$ has a unique finite fixed point $x_{2}$. In the latter case, one of the relations $(5.2)$ and

$$
\varphi(x+1)<\varphi(x)+1
$$

holds for $x<\varphi\left(x_{2}\right)$, and the other one holds for $x>\varphi\left(x_{2}\right)$.

5.2.3. Sublemma 3. There is nofunction $\varphi$ in $\mathscr{F}$ such that $\varphi(x)-x$ is bounded above for all $x$. Hence $\varphi(x)-x \rightarrow \infty$ as $x \rightarrow \infty$. 
Proof. To get a contradiction, suppose that there is a function $\varphi$ in $\mathscr{F}$ such that $\varphi(x)<x+M$ for all $x$. For an integer $k$, we write

$$
\varphi_{k}=T_{k} \circ \varphi \circ T_{-k} .
$$

Then

$$
x<\varphi_{k}(x)<x+M \text { for all } x \text { and } k .
$$

We have $\varphi_{k+1}(x)<\varphi_{k}(x)$ if and only if $\varphi(x-k-1)+1<\varphi(x-k)$. Hence the sequence $\varphi_{k}(x)$ is eventually monotonic for each $x$ as $k \rightarrow \infty$ and as $k \rightarrow-\infty$, and the limits

$$
\varphi_{+}(x)=\lim _{k \rightarrow \infty} \varphi_{k}(x) \text { and } \varphi_{-}(x)=\lim _{k \rightarrow-\infty} \varphi_{k}(x)
$$

exist for all $x$. In each case we may first pass to a subsequence and assume that (i) or (ii) in the definition of a convergence group holds for this subsequence and hence, by monotonicity, for the whole sequence $\varphi_{k}$. By (5.5), the alternative (ii) in that definition cannot occur, and so $\varphi_{+}$and $\varphi_{-}$are homeomorphisms and hence elements of $G$. Clearly $\varphi_{+}$and $\varphi_{-}$commute with $T_{1}$, and $x \leq \varphi_{+}(x)$, $\varphi_{-}(x) \leq x+M$ for all $x$.

Next we show that

$$
\varphi_{+}(x)<\varphi_{-}(x) \text { for all } x \text {. }
$$

We have $\varphi(x)<x+1$ for $x<x_{1}$ and $\varphi(x)>x+1$ for $x>x_{1}$, for some $x_{1}$. If $x$ is fixed, then eventually $x-k<x_{1}$ as $k \rightarrow \infty$, and $x-k>x_{1}$ as $k \rightarrow-\infty$. Hence

$$
\varphi_{k}(x)=\varphi(x-k)+k<(x-k)+1+k=x+1
$$

for $k>k_{1}(x)>0$ and $\varphi_{k}(x)>x+1$ for $k<k_{2}(x)<0$, say. Thus $\varphi_{+}(x) \leq$ $x+1 \leq \varphi_{-}(x)$ for all $x$. Since $\varphi_{+}, \varphi_{-}$, and $T_{1}$ belong to $G$, it follows from Lemma 2 that either (5.7) holds or $\varphi_{+}=\varphi_{-}=T_{1}$.

If $\varphi_{+}=T_{1}$, consider $\omega=T_{-1} \circ \varphi \in G$. We have $\omega(x)>x$ for $x>$ $x_{1}$ and $\omega(x)<x$ for $x<x_{1}$. If $\varepsilon>0$ is given, choose $k_{0}$ so large that $\left|\varphi_{k}(x)-T_{1}(x)\right|<\varepsilon$ if $k \geq k_{0}$ and $0 \leq x \leq 1$. With $y=x-k \leq 1-k_{0}$ this means that $|\omega(y)-y|<\varepsilon$. Hence $\omega(y)-y \rightarrow 0$ as $y \rightarrow-\infty$. Now Sublemma 1 gives a contradiction. So (5.7) holds, as asserted.

We have $\varphi^{*}=\varphi_{-} \circ \varphi_{+}^{-1} \in G$ and $\varphi^{*}(x)>x$ for all $x$. Further, we have

$$
m=\min \left\{\varphi^{*}(x)-x: 0 \leq x \leq 1\right\}>0 .
$$

Since $\varphi^{*}$ commutes with $T_{1}$, that is,

$$
\varphi^{*}(x+1)-(x+1)=\varphi^{*}(x)-x,
$$

we have $\varphi^{*}(x) \geq x+m$ for all $x$. Thus $\left(\varphi^{*}\right)^{p}(x) \geq x+p m$ for all $x$ if $p \geq 1$.

We fix an integer $p$ with $p>1 / m$ and note that $\left(\varphi_{-n} \circ \varphi_{n}^{-1}\right)^{p} \rightarrow\left(\varphi^{*}\right)^{p}$ uniformly on $[0,1]$ as $n \rightarrow \infty$. Hence if $n \geq n_{0}$, say, and $0 \leq x \leq 1$, we have

$$
\left|\left(\varphi^{*}\right)^{p}(x)-\left(\varphi_{-n} \circ \varphi_{n}^{-1}\right)^{p}(x)\right|<(p m-1) / 2
$$


and thus

$$
\begin{aligned}
\left(\varphi_{-n} \circ \varphi_{n}^{-1}\right)^{p}(x) & >\left(\varphi^{*}\right)^{p}(x)-(p m-1) / 2 \\
& \geq x+p m-(p m-1) / 2 \\
& =x+(p m+1) / 2>x+1 .
\end{aligned}
$$

Fix $n \geq n_{0}$. For any $x \in \mathbf{R}$, we have $x=y+N$ where $N \in \mathbf{Z}$ and $0 \leq y<1$. We have

$$
\left|\varphi_{n}(x)-\varphi_{-n}(x)\right|=\left|\varphi_{n-N}(y)-\varphi_{-n-N}(y)\right|<\varepsilon
$$

if $|N| \geq N_{0}$, say, that is, if $|x| \geq x_{0}$. Taking $x=\varphi_{n}^{-1}(z)$, so that $|x| \geq x_{0}$ if $z \geq \varphi_{n}\left(x_{0}\right)$ or if $z \leq \varphi_{n}\left(-x_{0}\right)$, we get

$$
\left|\left(\varphi_{-n} \circ \varphi_{n}^{-1}\right)(z)-z\right|<\varepsilon
$$

for such $z$. Hence $\left(\varphi_{-n} \circ \varphi_{n}^{-1}\right)(z)-z \rightarrow 0$ as $z \rightarrow \pm \infty$. Consequently, we have

$$
\lim _{|z| \rightarrow \infty}\left(\left(\varphi_{-n} \circ \varphi_{n}^{-1}\right)^{q}(z)-z\right)=0
$$

for each $q \geq 1$.

Now (5.9) with $q=p$ and (5.8) imply that the graphs of the distinct functions $T_{1}$ and $\left(\varphi_{-n} \circ \varphi_{n}^{-1}\right)^{p}$ have at least two finite points of intersection, which contradicts Lemma 2.

So $\varphi(x)-x$ cannot be bounded above, and thus for each $n \geq 1$, there is a point $y_{n}$ such that $\varphi\left(y_{n}\right)=y_{n}+n$ while $\varphi(x)<x+n$ for $x<y_{n}$. Since $T_{n} \in G$, it follows from Lemma 2 that $\varphi(x)>x+n$ for $x>y_{n}$. Thus $\varphi(x)-x \rightarrow \infty$ as $x \rightarrow \infty$. This proves Sublemma 3 .

5.2.4. Sublemma 4. If $\varphi \in \mathscr{F}$ then there is $x_{0}$ such that (5.2) holds for all $x>x_{0}$.

Proof. Suppose that this is false for some $\varphi \in \mathscr{F}$. Then by Sublemma 2, the function $F(\varphi)$ has a finite fixed point $x_{3}$ and (5.3) holds for $x>x_{4}$ while (5.2) is true for all $x<x_{4}$ where $x_{4}=\varphi\left(x_{3}+1\right)$. With $\psi(x)=\varphi(x)-x,(5.3)$ reads $\psi(x+1)<\psi(x)$ for $x>x_{4}$. Hence $\varphi(x)-x$ is bounded above, which contradicts Sublemma 3. This proves Sublemma 4.

In fact, one can prove that (5.2) holds for all $x$, that is, $F(\varphi)(x)>x$ for all $x$.

Sublemma 5. If $\varphi \in \mathscr{F}$ and $q \geq 1$, then there is $x_{q}$ such that

$$
F(\varphi)^{q}(x)<\varphi(x) \text { for all } x>x_{q} .
$$

Proof. We have $F(\varphi)(x)<\left(\varphi \circ T_{1} \circ \varphi^{-1}\right)(x)$ and hence

$$
F(\varphi)^{q}(x)<\left(\varphi \circ T_{q} \circ \varphi^{-1}\right)(x) \text { for all } x .
$$


Thus (5.10) holds provided that, with $x=\varphi(y)$, we have $\varphi(y+q)<\varphi^{2}(y)$, that is, $y+q<\varphi(y)$. By Sublemma 3, this is true for all large $y$, and Sublemma 5 is proved.

In fact one can prove that $F(\varphi)(x)<\varphi(x)$ for all $x$. This is not true for $\varphi(x)=x+e^{x}$, which then implies that $x+1$ and $x+e^{x}$ do not generate a convergence group. However, for $\varphi(x)=x+2^{x}$, for example, we do not get a contradiction in this way, considering only $q=1$.

5.2.5. To prove Lemma 6, pick any $\varphi \in \mathscr{F}$, define $\varphi_{k}$ by $(5.4)$ and $\varphi_{+}$by (5.6). As in the proof of Sublemma 3 we deduce that $\varphi_{+}$exists and belongs to $G$. We set $g=\varphi \circ \varphi_{+}^{-1} \in G$. The definition of $\varphi_{+}$implies that $g(x)-x \rightarrow 0$ as $x \rightarrow-\infty$, so that $g(x)<x+1$ for $x<x_{5}$, say. Since $\varphi_{+}$commutes with $T_{1}$, the function $\varphi_{+}(x)-x$ is bounded. Hence by Sublemma 3, we have $g(x)-x \rightarrow \infty$ as $x \rightarrow \infty$. Finally, $g(x)>x$ for all $x$. For if not, then $g(x)<x$ for $x<x_{6}$, say, and by what was proved above, we may apply Sublemma 1 to $\omega=g$ and obtain a contradiction. Hence $g \in \mathscr{F}$.

As $x \rightarrow-\infty$, we have $g(x)-x \rightarrow 0$, and hence $F(g)(x)-x \rightarrow 0$. Consequently, $F(g)^{q}(x)-x \rightarrow 0$ also, for each $q \geq 1$. Thus $h_{q}=g \circ F(g)^{-q}$ is in $G$ and satisfies

$$
\lim _{x \rightarrow-\infty}\left(h_{q}(x)-x\right)=0
$$

for each $q \geq 1$.

By Sublemma 5, we have $h_{q}(x)>x$ for all large $x$. By Sublemma 4, there is $y$ such that $F(g)(x)>x$ for $x>y$. Hence $F(g)^{q}(x) \rightarrow \infty$ as $q \rightarrow \infty$ whenever $x>y$. So for $z=y+1$, there is $q \geq 1$ such that $F(g)^{q}(z)>g(z)$. With $z_{1}=F(g)^{q}(z)$, this reads $z_{1}>h_{q}\left(z_{1}\right)$. Now it follows from Lemma 2 that there is $z_{2}$ such that $h_{q}(x)<x$ for all $x<z_{2}$. In view of this and (5.11), we may apply Sublemma 1 to $\omega=h_{q}$ and obtain a contradiction. Thus the class $\mathscr{F}$ is empty, and the first statement of Lemma 6 is proved.

It is now obvious that if $g, h \in G_{T}$ then $g^{-1} \circ h \in G_{T}$ so that $G_{T}$ is a subgroup of $G$. Also $G_{T}$ is a convergence group since $G$ is. This proves Lemma 6.

5.3. Proof of Lemma 9. First we note that $H \neq$ Id, for if $H=$ Id then $g \circ h=$ $h \circ g$. But then, if $g$ fixes $x_{1}$ and $h$ fixes $x_{2}$, we get $g\left(x_{2}\right)=h\left(g\left(x_{2}\right)\right)$ so that $g\left(x_{2}\right)=x_{2}$ since $x_{2}$ is the unique finite fixed point of $h$, by Lemma 1 . This implies similarly that $x_{1}=x_{2}$, which is against our assumption, and so $H \neq \mathrm{Id}$. Actually, this argument should have been given at the beginning of the proof of [2, Lemma 6], but it was omitted there.

By passing to inverse functions, if necessary, we may assume that $g(x)>x$ and $h(x)>x$ for all large $x$. By interchanging $g$ and $h$, if necessary, we may assume that there is $x_{1}$ such that $g(x)<h(x)$ for $x>x_{1}$ (and possibly for all $x$, of course). By performing a preliminary conjugation, we may then 
assume that $h(x)=2 x$. Then $g$ fixes $x_{2}$ where $x_{2} \neq 0$, and $g(x)>x$ for $x>x_{2}$. To see that this does not change the problem note that if, for example, $g$ is replaced by $g^{-1}$, then we need to consider

$$
g^{-1} \circ h \circ g \circ h^{-1}=g^{-1} \circ H^{-1} \circ g=H_{1},
$$

say. If the roles of $g$ and $h$ are interchanged, we consider $h \circ g \circ h^{-1} \circ g^{-1}=$ $H^{-1}$. But $H$ has a finite fixed point if and only if $H^{-1}$ or $H_{1}$ has such a fixed point.

5.3.1. Suppose that $H$ has a finite fixed point, where $H(x)=g\left(2 g^{-1}(x / 2)\right)$. Then the graphs of $g(2 x)$ and $2 g(x)$ intersect at a finite point $x_{3}$. We assume that

$$
g(2 x)>2 g(x) \text { for } x>x_{3} .
$$

By Lemma 2, we then have

$$
g(2 x)<2 g(x) \text { for } x<x_{3} .
$$

For $n \geq 0$, we define $g_{n} \in G$ by $g_{n}(x)=2^{-n} g\left(2^{n} x\right)$. By (5.12), we have

$$
g_{n+1}(x)>g_{n}(x) \text { if and only if } x>2^{-n} x_{3},
$$

and $2^{-n} x_{3} \rightarrow 0$ as $n \rightarrow \infty$. There is $x_{4}>0$ such that $g(x)<h(x)=2 x$ for $x>x_{4}$, and thus $g_{n}(x)<2 x$ for $x>x_{4}$. Since $g_{n}(x)$ is eventually monotonic for any fixed nonzero $x$ by (5.14), and trivially for $x=0$, and since $G$ is a convergence group, we deduce that $g_{n}(x) \rightarrow g_{+}(x)$ as $n \rightarrow \infty$, where $g_{+}$is a homeomorphism and $g(x)<g_{+}(x) \leq 2 x$ for $x>x_{4}$. Clearly $g_{+}(2 x)=2 g_{+}(x)$ for all $x$.

We may assume that $G$ is closed so that $g_{+} \in G$ and thus $g^{*}=g_{+} \circ g^{-1} \in G$. We have $g_{n}(x) \rightarrow g_{+}(x)$ as $n \rightarrow \infty$ uniformly on an interval [y,2y] where $y>0$ and $g_{+}(y) \geq 1$. Therefore

$$
\frac{g_{+}\left(2^{n} z\right)}{g\left(2^{n} z\right)}=\frac{2^{-n} g_{+}\left(2^{n} z\right)}{2^{-n} g\left(2^{n} z\right)}=\frac{g_{+}(z)}{g_{n}(z)} \rightarrow 1
$$

as $n \rightarrow \infty$ uniformly for $y \leq z \leq 2 y$. It follows that

$$
\lim _{x \rightarrow \infty} \frac{g^{*}(x)}{x}=\lim _{x \rightarrow \infty} \frac{g_{+}(x)}{g(x)}=1 .
$$

Hence

$$
\lim _{x \rightarrow \infty} \frac{\left(g^{*}\right)^{q}(x)}{x}=1 \text { for each } q \geq 1 .
$$

For $x<\min \left(0, x_{3}\right)$, we have $g\left(2^{n} x\right)<2^{n} g(x)$ for all $n \geq 1$. Hence $g_{n}(x)<g(x)$ and so $g_{+}(x) \leq g(x)$ for these $x$. Since $g_{+}(x)>g(x)$ for $x>x_{4}$, it follows from Lemma 2 that $g^{*}$ has a finite fixed point $x_{5}$ and that $g^{*}(x)>x$ if and only if $x>x_{5}$. 
Since $\left(g^{*}\right)^{q}\left(x_{5}+1\right) \rightarrow \infty$ and $\left(g^{*}\right)^{q}\left(x_{5}-1\right) \rightarrow-\infty$ as $q \rightarrow \infty$, we conclude that for a sufficiently large $q$, we have

$$
\left(g^{*}\right)^{q}\left(x_{5}+1\right)>2\left(x_{5}+1\right) \text { and }\left(g^{*}\right)^{q}\left(x_{5}-1\right)<2\left(x_{5}-1\right) \text {. }
$$

By this and (5.15), the graphs of the distinct elements $\left(g^{*}\right)^{q}$ and $M_{2}$ of $G$ have at least two finite points of intersection, which gives a contradiction by Lemma 2. If, instead of (5.12), we have $g(2 x)<2 g(x)$ for $x>x_{3}$, then an analogous argument gives a contradiction. This proves Lemma 9.

\section{FunCtions WITH A COMMON FIXED POINT}

6.1. In this section we shall prove case (i) of Theorem 1 . We prefer to state our intermediate results as lemmas to make it easier to refer to them.

Lemma 10. If all the elements of the closed noncyclic convergence group $G$ have a common fixed point, then there is a homeomorphism $f$ such that $f^{-1} \circ G \circ f$ is a Möbius group.

We may and will assume that $G$ is defined on $\overline{\mathbf{R}}$ and that the common fixed point is the point at infinity. First we consider the case when all the functions in $G$ are increasing.

Lemma 11. If all the elements of the closed noncyclic convergence group $G$ are increasing functions on $\mathbf{R}$ and fix infinity, then there is an increasing homeomorphism $f$ of $\overline{\mathbf{R}}$ fixing infinity such that $f^{-1} \circ G \circ f$ is a group of functions of the form $a x+b$ where $a>0$ and $b$ is real.

6.1.1. We prove the following result, after which the rest can be done with less difficulty.

Lemma 12. If $G=G_{T} \neq\{\mathrm{Id}\}$ is closed and not cyclic, then there is a homeomorphism $f$ such that $f^{-1} \circ G \circ f=\mathscr{T}$, the group of all translations of $\mathbf{R}$.

Proof. To prove Lemma 12, we may proceed as in [2, pp. 335-336] when proving the case $G=G_{T}$ of [2, Lemma 10]. The convergence properties required for Sublemma 1 in [2, p. 335] now follow from the fact that $G$ is a convergence group. In this way we may establish that $G(x)=\{g(x): g \in G\}=\mathbf{R}$ for all $x \in \mathbf{R}$ and that for each $g \in G$, there is $h \in G$ such that $g=h^{2}(=h \circ h)$.

At this point we must essentially deviate from the proof in [2, p. 336] since the functions $f_{i}$ that would conjugate various cyclic subgroups of $G$ to Möbius groups are now only homeomorphisms, not necessarily quasisymmetric, and thus not known to form a normal family. Therefore we cannot find the homeomorphism $f$ for $G$ as a limit of the $f_{i}$, but must rather define $f$ in one go, which we now proceed to do.

We choose $g_{0} \in G$ with $g_{0}(x)>x$ for all $x$, and agree that $g_{0}$ is to be conjugated to $T_{1}$. For $n \geq 1$, we find functions $g_{n} \in G$ such that $g_{n}^{2}=g_{n-1}$. We may choose $f(0)$ in an arbitrary way; we take $f(0)=0$. For $x=j / 2^{n}$ 
where $j$ is an integer and $n \geq 0$, we set $f(x)=g_{n}^{j}(0)$. Since $g_{m}$ is an iterate of $g_{n}$ if $n>m$, it follows that the $g_{n}$ commute. Hence $f(x)$ does not depend on the representation of $x$ in the form $j / 2^{n}$. If $x_{1}$ and $x_{2}$ are dyadic rational numbers and $x_{1}<x_{2}$, then we may write $x_{1}=j / 2^{n}$ and $x_{2}=k / 2^{n}$ where $j<k$, so that

$$
f\left(x_{1}\right)=g_{n}^{j}(0)<g_{n}^{k}(0)=f\left(x_{2}\right) .
$$

(To see this, note that each $g_{n}$ can be conjugated to a translation such as $T_{1}$.) Thus $f$ is strictly increasing on the set $S=\left\{j 2^{-n}: j \in \mathbf{Z}, n \geq 0\right\}$ so that if $f$ is continuous on $S$, then $f$ has a unique extension to an increasing homeomorphism of $\overline{\mathbf{R}}$ fixing infinity. Note here also that $f(x) \rightarrow \infty$ as $x \rightarrow \infty$ in $S$ since, for example, $g_{0}^{k}(0) \rightarrow \infty$ as $k \rightarrow \infty$. Similarly, $f(x) \rightarrow-\infty$ as $x \rightarrow-\infty$ in $S$.

6.1.2. To see that $f$ is continuous at any $x \in S$, we use the following argument. For simplicity, we present the details only for right-hand continuity at $x=0$. Suppose that $x_{n}$ is a strictly decreasing sequence of elements of $S$ tending to zero. Then $0<f\left(x_{n+1}\right)<f\left(x_{n}\right)$ for all $n$, so that $f\left(x_{n}\right) \rightarrow a \geq 0$ as $n \rightarrow \infty$. We need to show that $a=f(0)=0$. With each $x_{n}=k / 2^{m}$ we associate the function $h_{n}=g_{m}^{k} \in G$, so that $f\left(x_{n}\right)=h_{n}(0)$. By passing to a subsequence, if necessary, we may assume that $h_{n} \rightarrow h \in G$. (Since $x<h_{n}(x) \leq h_{1}(x)$ for all $x$ and all $n \geq 1$, possibility (ii) in the definition of a convergence group cannot occur.) If $a>0$, then $a=\lim _{n \rightarrow \infty} h_{n}(0)=h(0)$, and so $h(x)>x$ for all $x$ by Lemma 2 .

For any $N \geq 1$, there is $x_{n}$ with $x_{n}=k / 2^{m}<1 / N$. Then $h^{N}(x) \leq h_{n}^{N}(x) \leq$ $g_{0}(x)$ for all $x$. But $h^{N}(0) \rightarrow \infty$ as $N \rightarrow \infty$, which is a contradiction. Hence $a=0$, as required.

6.1.3. We claim that $f^{-1} \circ g_{n} \circ f=T_{2-n}$ for all $n \geq 0$. Then $G_{1}=f^{-1} \circ G \circ f$ contains the subgroup $\left\{T_{s}: s \in S\right\}$, and so by Lemma 8, the group $G_{1}$ is a Möbius group. Since $G_{1}$ is closed and each function in $G_{1} \backslash\{\mathrm{Id}\}$ has no finite fixed points, we conclude that $G_{1}=\mathscr{T}$. This will then prove Lemma 12 .

Since $f$ and $g_{n}$ are continuous, it suffices to show that

$$
g_{n}(f(x))=f\left(x+2^{-n}\right) \text { for all } x \in S .
$$

If $x=k / 2^{m} \in S$ then we may choose the representation for $x$ so that $m \geq n$. This gives

$$
g_{n}(f(x))=g_{m}^{2^{m-n}}\left(g_{m}^{k}(0)\right)=g_{m}^{M}(0)
$$

where $M=k+2^{m-n}$, while $x+2^{-n}=M / 2^{m}$ and so $f\left(x+2^{-n}\right)=g_{m}^{M}(0)$ also. This proves (6.1), and the proof of Lemma 12 is complete.

6.2. We continue the proof of Lemma 11. In view of Lemma 12, we may assume that $G \neq G_{T}$. Suppose that $G \backslash\{\operatorname{Id}\}$ contains two elements with distinct finite fixed points. Then $G_{T} \neq\{$ Id $\}$ by Lemma 9 . Next, by Lemma 7 we see that 
$G_{T}$ is closed and not cyclic, so that by Lemma 12 there is a homeomorphism $f$ such that $f^{-1} \circ G_{T} \circ f=\mathscr{T}$. Since $G_{1}=f^{-1} \circ G \circ f$ contains $\mathscr{T}$, it follows from Lemma 8 that $G_{1}$ is a Möbius group.

Hence we may assume that all functions in $G \backslash G_{T}$ have a common finite fixed point, which we may take to be the origin. If $G_{T} \neq\{\mathrm{Id}\}$, we may argue as above, so we assume that $G_{T}=\{\mathrm{Id}\}$. Thus all the elements of $G$ fix 0 and $\infty$. Since $G$ is closed and not cyclic, we may proceed as in the proof of [2, Lemma 10, pp. 336-337] to show that for any $g \in G$ there is $h \in G$ with $h^{2}=g$.

We now continue as in the proof of Lemma 12 in Subsection 6.1 and choose $g_{0} \in G$ with $g_{0}(x)>x$ for $x>0$ that we conjugate to $M_{e}$. Recall that $M_{s}(x)=s x$. We find $g_{n} \in G$ such that $g_{n}^{2}=g_{n-1}$ for $n \geq 1$. If $x>0$ and $\log x=k / 2^{n}$ where $k \in \mathbf{Z}$ and $n \geq 0$, we set $f(x)=g_{n}^{k}(1)$. If $x<0$ and $\log (-x)=k / 2^{n}$, we set $f(x)=g_{n}^{k}(-1)$. Hence $f(1)=1$ and $f(-1)=-1$. As before, we can show that $f$ is strictly increasing and continuous on the dense subset $S_{1}=\left\{x: \log |x|=k / 2^{n}, k \in \mathbf{Z}, n \geq 0\right\}$ of $\mathbf{R}$ and that $f$ extends to an increasing homeomorphism of $\overline{\mathbf{R}}$ fixing 0 and $\infty$. Furthermore, we have $\left(f^{-1} \circ g_{n} \circ f\right)(x)=x \exp \left(2^{-n}\right)$, first for all $x \in S_{1}$ and thus for all $x \in \overline{\mathbf{R}}$, for each $n \geq 0$. Hence the group $G_{2}=f^{-1} \circ G \circ f$ contains the subgroup $\left\{M_{t}: \log t=k / 2^{n}, k \in \mathbf{Z}, n \geq 0\right\}$. Since $G_{2}$ is closed, we have $\mathscr{M} \subset G_{2}$. If $h \in G_{2}$, there is $b>0$ such that $h(x)=M_{b}(x)=b x$ for $x=0,1$, and $\infty$. Thus $h=M_{b}$ by Lemma 2, and so $G_{2}=\mathscr{M}$ is a Möbius group. This completes the proof of Lemma 11.

Remark. Another approach to the case $G_{T}=\{\mathrm{Id}\}$ would be to reduce it to two applications of Lemma 12 by considering the groups $G_{1}=L_{1} \circ G \circ L_{1}^{-1}$ and $G_{2}=L_{2} \circ G \circ L_{2}^{-1}$, which are conjugate to $\mathscr{T}$, where $L_{1}(x)=\log x$ for $x>0$ and $L_{2}(x)=\log (-x)$ for $x<0$. In this way we find a homeomorphism $f$ of $\overline{\mathbf{R}}$ such that for each $g \in G$, the function $f^{-1} \circ g \circ f$ is of the form $a(g) x$ for $x>0$ and $b(g) x$ for $x<0$, for some positive $a(g)$ and $b(g)$, with $a(g)=b(g)$ for at least one preassigned $g \in G \backslash\{$ Id $\}$. However, doing this and proving carefully that then $a(g)=b(g)$ for all $g \in G$ so that $f^{-1} \circ G \circ f=\mathscr{M}$ seems to lead to a longer presentation than the proof given above.

6.3. To complete the proof of Lemma 10, we may, in view of Lemma 11, perform a preliminary conjugation and assume that the subgroup $G_{0}$ consisting of the increasing functions in $G$ is a Möbius group and that $G \neq G_{0}$. Now we may proceed as in $[3, \S 3]$ to identify the five cases that can occur and to construct $f$ in each case. Nothing needs to be changed, but now it will suffice to prove only that $f$ is a homeomorphism, not necessarily quasisymmetric. For completeness, we briefly review the five possible cases that arise, sometimes after a further preliminary conjugation of $G$ by a function of the form $a x+b$ :

(1) $G_{0}$ contains the group $\mathscr{T}$ of all translations. In this case $G$ is already a Möbius group, by Lemma 8 given in $\S 5$ above. 
(2) $G_{0}=\{$ Id $\}$. Then $G=\{g$, Id $\}$ where $g \circ g=$ Id. The decreasing homeomorphism $g$ of $\mathbf{R}$ has a unique finite fixed point, which we may assume to be the origin. We set $f(x)=-g(x)$ for $x \leq 0$ and $f(x)=x$ for $x>0$. Then $f$ is an increasing homeomorphism of $\mathbf{R}$ and $\left(f \circ g \circ f^{-1}\right)(x)=-x$, so that $f \circ G \circ f^{-1}$ is a Möbius group.

(3) $G_{0}=\mathscr{M}$, the group of all increasing dilations of $\mathbf{R}$. Now it is shown as in [3, pp. 12-13] that $G$ is generated by $\mathscr{M}$ and a single function $g$ in $G \backslash G_{0}$, and further that any such $g$ is of the form $g(x)=-A x$ for $x \leq 0$ and $g(x)=-B x$ for $x>0$ for some positive numbers $A$ and $B$. We set $f(x)=x$ for $x \leq 0$ and $f(x)=\sqrt{B / A} x$ for $x>0$. Then $\left(f \circ g \circ f^{-1}\right)(x)=-\sqrt{A B} x$ while $f \circ M_{b} \circ f^{-1}=M_{b}$ for all $b>0$. Thus $f \circ G \circ f^{-1}$ is a Möbius group.

(4) $G_{0}$ is the cyclic group generated by the translation $T_{1}$. Now $G$ is generated by $G_{0}$ and a fixed function $g \in G \backslash G_{0}$. As in [3, subsection 3.5], one shows that $g(x+1)=g(x)-1$ for all real $x$. The decreasing function $g$ has a unique finite fixed point, say the origin. Since $g(x)-x$ is strictly decreasing and continuous on $[0,1]$ and takes the values 0 and -2 at the endpoints, there is a unique point $x_{1} \in(0,1)$ with $g\left(x_{1}\right)=x_{1}-1<0$. We set $f(x)=x /\left(2 x_{1}\right)$ for $0 \leq x \leq x_{1}$ and $f(x)=-g^{-1}(x) /\left(2 x_{1}\right)$ for $g\left(x_{1}\right) \leq x<0$. Then $f$ is strictly increasing and continuous on $\left[g\left(x_{1}\right), g\left(x_{1}\right)+1\right]$ and satisfies

$$
f\left(g\left(x_{1}\right)+1\right)=f\left(x_{1}\right)=\frac{1}{2}=-\frac{1}{2}+1=f\left(g\left(x_{1}\right)\right)+1 .
$$

Now the condition $f(x+1)=f(x)+1$ extends $f$ to an increasing homeomorphism of $\mathbf{R}$ that conjugates $T_{1}$ onto itself and satisfies $\left(f \circ g \circ f^{-1}\right)(x)=-x$. Thus $f \circ G \circ f^{-1}$ is a Möbius group.

(5) $G_{0}$ is generated by the dilation $M_{b}$ where $b>1$. This corresponds to case (ii) in [3, §3]. As in [3, subsections 3.6 and 3.7], we see that there is $g \in G \backslash G_{0}$ fixing the origin and commuting with $M_{b}$ such that $G$ is generated by $g$ and $M_{b}$. We have $g^{2}(x)=b^{n} x$ for some integer $n$, and we set $a=b^{n / 2}$ (possibly $n=0$ ). We define $f(x)=x$ for $x \geq 0$ and $f(x)=-a^{-1} g(x)$ for $x<0$. Then $f$ is an increasing homeomorphism of $\mathbf{R}$ and $f \circ M_{b} \circ f^{-1}=M_{b}$ while $f \circ g \circ f^{-1}=M_{-a}$. Thus $f \circ G \circ f^{-1}$ is a Möbius group.

This proves Lemma 10.

Now case (i) of Theorem 1 follows from Lemmas 4, 5, and 10.

\section{FINITE GROUPS}

7.1. In this section we prove case (iii) of Theorem 1 as well as Theorem 2 . Let $G$ be a finite convergence group on $\mathbf{T}$. By considering the possibilities that can arise in view of Lemma 4, we see that each element in the subgroup $G_{0}$ of sense-preserving elements of $G$ can be conjugated to a rotation $R_{q}$ where $q$ is a rational number, and that each sense-reversing element $g$ of $G$ satisfies $g^{2}=$ Id. If $g, h \in G \backslash G_{0}$ then $h \circ g^{-1}=h_{1} \in G_{0}$ and so $h=h_{1} \circ g$. Hence 
either $G=G_{0}$ or

$$
G=G_{0} \cup\left\{h \circ g: h \in G_{0}\right\} \text { for some } g \in G \backslash G_{0},
$$

and any $g \in G \backslash G_{0}$ would do..

The most essential part of the proof is to show that $G_{0}$ is abelian, which then implies that $G_{0}$ is cyclic, as we shall see.

Lemma 13. If $g$ and $h$ are sense-preserving elements of the finite convergence group $G$, then $g \circ h=h \circ g$.

Proof. We may assume that $g \neq h$ and that $g \neq \mathrm{Id} \neq h$. We may represent $g$ by a map $g_{0}$ of $\mathbf{R}$ onto itself such that $0<g_{0}(0)<1$,

$$
g_{0}(x+1)=g_{0}(x)+1 \text { and } e\left(g_{0}(x)\right)=g(e(x))
$$

for all real $x$. Similarly, we may represent $h$ by $h_{0}$. In view of Lemma 4 , we may perform a preliminary conjugation of $G$ and assume that $g$ is a rotation. Then $g_{0}(x)=x+q$ where $q$ is a rational number on $(0,1)$ while $x<h_{0}(x)<x+1$ for all real $x$. (Recall that $h$ has no fixed points on $\mathbf{T}$.)

Suppose that $g \circ h \neq h \circ g$. Then $H=g \circ h \circ g^{-1} \circ h^{-1} \in G_{0} \backslash\{$ Id $\}$, so that $H$ has no fixed points on $\mathrm{T}$ and $H$ is of finite order. We represent $g \circ h$ by $h_{0}(x)+q=h_{1}(x)$, say, and $h \circ g$ by $h_{0}(x+q)=h_{2}(x)$. It does not matter if $h_{1}(0) \geq 1$ or $h_{2}(0) \geq 1$. The graphs of $h_{1}$ and $h_{2}$ cannot intersect, and so we have, for example, $h_{1}(x)<h_{2}(x)$ for all $x$. Then

$$
h_{0}(x)+q<h_{0}(x+q)
$$

and hence

$$
h_{0}(x)+n q<h_{0}(x+n q)
$$

for all real $x$ and all positive integers $n$. Choosing $n$ so that $n q$ is an integer, we get a contradiction since $h_{0}(x+m)=h_{0}(x)+m$ for all $x \in \mathbf{R}$ and $m \in \mathbf{Z}$. This proves Lemma 13.

7.2. Since $G_{0}$ is finite, it contains an element $g$ of maximal order. Since, furthermore, $G_{0}$ is abelian by Lemma 13, we may follow the proof of [3, Lemma 7] and deduce that $g$ generates $G_{0}$. Even though [3, Lemma 7] is formulated for $K$-quasisymmetric groups, the proof works without changes for convergence groups also. This together with (7.1) proves that $G$ has the structure given by (1.1) or (1.2).

Let $g$ generate $G_{0}$. By Lemma 4, and by replacing $g$ by a suitable iterate of $g$ that also generates $G_{0}$, we find a homeomorphism $f_{1}$ such that $f_{1} \circ g \circ f_{1}^{-1}=$ $R_{1 / N}$ where $N$ is the order of $g$. If $G$ is a $K$-quasisymmetric group, we may take $f_{1}$ to be $K_{1}$-qs where $K_{1}$ depends on $K$ only, by [3, Theorem 2]. We write $f_{1} \circ G \circ f_{1}^{-1}=G_{1}$ and $f_{1} \circ G_{0} \circ f_{1}^{-1}=G_{2}$ so that $G_{2}$ is generated by $R_{1 / N}$. If $G$ is $K$-qs then $G_{1}$ is $K_{2}$-qs where $K_{2}$ depends on $K$ only. 
7.3. It remains to consider the case when $G \neq G_{0}$ so that $G_{1}=G_{2} \cup\left\{R_{m / N} \circ\right.$ $h: 1 \leq m \leq N\}$ where $h$ is sense-reversing and $h^{2}=$ Id. We may and will assume that $N \geq 2$, for otherwise $G_{1}$ and hence $G$ is a cyclic group of order two, generated by a sense-reversing function, and therefore topologically conjugate to a Möbius group by Lemma 4 . For brevity, we write $g_{m}=R_{m / N}$. Since $h \circ g_{1} \circ h^{-1} \in G_{2}$, we have $h(c z)=c^{-l} h(z)$ for some $l$ with $1 \leq l \leq N$, where $c=e(1 / N)$. Since $h \circ g_{1} \in G_{1} \backslash G_{2}$, we have $\left(h \circ g_{1}\right)^{2}=\mathrm{Id}$, and so $z=h(c h(c z))=c^{-l} h^{2}(c z)=c^{1-l} z$. Hence $l=1$ and

$$
h(c z)=\bar{c} h(z) \text { for all } z \in \mathbf{T} .
$$

We look for a homeomorphism $f$ such that

$$
f(c z)=c f(z)
$$

and

$$
f(h(z))=\overline{f(z)} \text { for all } z \in \mathbf{T} .
$$

Then $f \circ g_{1} \circ f^{-1}=g_{1}$ and $\left(f \circ h \circ f^{-1}\right)(z)=\bar{z}$ so that $f \circ G_{1} \circ f^{-1}$ is a Möbius group. If $G_{1}$ is $K$-qs (denoting $K_{2}$ by $K$ again, for simplicity), we want to make $f K_{1}$-qs where $K_{1}$ depends on $K$ only.

The function $h$ has exactly two fixed points on $\mathbf{T}$. We may conjugate $G_{1}$ by a rotation, if necessary, and assume that $h(1)=1$.

Recall the notation (2.1). Since $h$ is sense-reversing with $h(1)=1$ and $h(c)=\bar{c} h(1)=\bar{c}$, there is a unique point $z_{1}=e\left(\theta_{1}\right)$ where $0<\theta_{1}<1 / N$ such that $h\left(z_{1}\right)=\bar{c} z_{1}$. (Note that the function $h_{0}$ representing $h$ on $\mathbf{R}$ is decreasing with $h_{0}(0)=0$ and $h_{0}(1 / N)=-1 / N$ while $\tilde{h}(x)=x-1 / N$ is increasing with $\tilde{h}(0)=-1 / N$ and $\tilde{h}(1 / N)=0$.) For $0 \leq \theta \leq \theta_{1}$, we define $f(e(\theta))=e\left(\theta\left[2 N \theta_{1}\right]^{-1}\right)$. For $z=e(\theta)$ where $\theta_{1}-1 / N \leq \theta<0$, we define $f(z)=\overline{f(h(z))}$, in accordance with (7.4). Then $f\left(z_{1}\right)=e\left([2 N]^{-1}\right)=d$, say, and

$$
f\left(\bar{c} z_{1}\right)=\overline{f\left(h\left(\bar{c} z_{1}\right)\right)}=\overline{f\left(h^{2}\left(z_{1}\right)\right)}=\overline{f\left(z_{1}\right)}=\bar{c} f\left(z_{1}\right) .
$$

Now we have defined $f$ on the arc $\left[\bar{c} z_{1}, z_{1}\right]$ with $f\left(z_{1}\right)=c f\left(\bar{c} z_{1}\right)$, and $f$ is a homeomorphism of $\left[\bar{c} z_{1}, z_{1}\right]$ onto $[\bar{d}, d]$. Hence (7.3) extends $f$ to a homeomorphism of $\mathbf{T}$ onto itself.

7.4. To check that $f$ satisfies (7.4) also, take $z \in \mathbf{T}$, and note that $z=c^{m} w$ where $w \in\left[\bar{c} z_{1}, z_{1}\right)$. Hence

$$
f(h(z))=f\left(\bar{c}^{m} h(w)\right)=\bar{c}^{m} f(h(w))=\overline{c^{m} f(w)}=\overline{f(z)}
$$

by (7.2) and (7.3) if (7.4) holds with $z$ replaced by $w$. If $w \in\left[\bar{c} z_{1}, 1\right)$ then (7.4) holds, with $z=w$, by the definition of $f$. If $w \in\left[1, z_{1}\right)$ then $w=h(u)$ where $u \in\left[\bar{c} z_{1}, 1\right)$ and so

$$
f(h(w))=f(u)=\overline{f(h(u))}=\overline{f(w)},
$$

as required. Thus (7.4) holds for all $z \in \mathbf{T}$. 
7.5. The above proves (iii) of Theorem 1 . To complete the proof of Theorem 2, we need to show that if $G_{1}$ is $K$-qs then the above $f$ is $K_{1}(K)$-qs. We choose a quadruple $Z=\left(z_{1}, z_{2}, z_{3}, z_{4}\right)$ of distinct points of $\mathbf{T}$ that follow each other in the positive direction such that $C(Z)=\frac{1}{2}$. We need to prove that then

$$
\left(2 K_{1}\right)^{-1} \leq C(f Z) \leq 1-\left(2 K_{1}\right)^{-1} .
$$

For the rest of the proof, we shall denote by $K_{1}$ any constant greater than 1 depending on $K$ only, not necessarily the same at every occurrence. Further, we denote by $\varepsilon_{0}, \varepsilon_{1}, \varepsilon_{2}, \ldots$ quantities in $\left(0, \frac{1}{2}\right]$ that depend on $K$ only. In cross-ratio calculations, note that

$$
\begin{aligned}
C\left(z_{1}, z_{2}, z_{3}, z_{4}\right) & =C\left(z_{3}, z_{4}, z_{1}, z_{2}\right) \\
& =1-C\left(z_{4}, z_{1}, z_{2}, z_{3}\right)=1-C\left(z_{2}, z_{3}, z_{4}, z_{1}\right) .
\end{aligned}
$$

To prove (7.5), let $f_{0}$ and $h_{0}$ represent $f$ and $h$ on $\mathbf{R}$, respectively, so that $e\left(f_{0}(x)\right)=f(e(x))$ and $e\left(h_{0}(x)\right)=h(e(x))$ for all real $x$. Since $f(1)=$ $h(1)=1$, we may take $f_{0}(0)=h_{0}(0)=0$. Then $f_{0}$ is strictly increasing, $h_{0}$ is strictly decreasing, and $f_{0}(x+1)=f_{0}(x)+1$ and $h_{0}(x+1)=h_{0}(x)-1$ for all $x \in \mathbf{R}$. By (7.2) and (7.3) we have the stronger identities

$$
f_{0}(x+1 / N)=f_{0}(x)+1 / N
$$

and

$$
h_{0}(x+1 / N)=h_{0}(x)-1 / N,
$$

valid for all real $x$. The relation $h\left(z_{1}\right)=\bar{c} z_{1}$ together with (7.8) implies that

$$
h_{0}\left(\theta_{1}\right)=\theta_{1}-N^{-1} \text { and } h_{0}\left(\theta_{1}-N^{-1}\right)=\theta_{1} .
$$

7.6. We shall soon prove that $f_{0}$ is $K_{1}$-qs on $\mathbf{R}$. Assuming this, consider a quadruple $Z$ on $\mathbf{T}$ with $C(Z)=\frac{1}{2}$. At least one of the arcs $\left(z_{i}, z_{i+1}\right)$, where $z_{5}=z_{1}$, has length at least $\pi / 2$. By (7.6), we may renumber the $z_{i}$, if necessary, and assume that $\left|\left(z_{4}, z_{1}\right)\right| \geq \pi / 2$. Suppose that $z_{i}=e\left(x_{i}\right)$ where $0 \leq x_{1}<1$ and $x_{1}<x_{2}<x_{3}<x_{4}<x_{1}+1$. In fact, then $x_{4} \leq x_{1}+\frac{3}{4}$. Thus

$$
A \leq C\left(x_{1}, x_{2}, x_{3}, x_{4}\right) \leq 1-A
$$

where $A$ is an absolute constant with $0<A \leq \frac{1}{2}$, as the reader may verify.

Suppose that $f_{0}$ is $K_{1}$-qs on $\overline{\mathbf{R}}$ with $f_{0}(\infty)=\infty$. Then

$$
0<\varepsilon_{0} \leq C\left(f_{0}\left(x_{1}\right), f_{0}\left(x_{2}\right), f_{0}\left(x_{3}\right), f_{0}\left(x_{4}\right)\right) \leq 1-\varepsilon_{0}<1 .
$$

We may deduce from this that

$$
0<\varepsilon_{1} \leq C(f Z) \leq 1-\varepsilon_{1}<1,
$$

provided that

$$
\left(f_{0}\left(x_{1}\right)+1\right)-f_{0}\left(x_{4}\right) \geq \varepsilon_{2}\left(f_{0}\left(x_{4}\right)-f_{0}\left(x_{1}\right)\right) .
$$


Since $f_{0}\left(x_{1}\right)+1=f_{0}\left(x_{1}+1\right)$, since $x_{4} \leq x_{1}+\frac{3}{4}$, so that $x_{4}-x_{1} \leq 3\left[\left(x_{1}+1\right)-x_{4}\right]$, and since $f_{0}$ is $K_{1}$-qs, standard distortion theorems for qs functions imply that (7.11) and hence (7.10) holds.

It remains to prove that $f_{0}$ is $K_{1}$-qs on $\overline{\mathbf{R}}$, which will be the case provided that

$$
\frac{1}{K_{1}} \leq \frac{f_{0}(x+t)-f_{0}(x)}{f_{0}(x)-f_{0}(x-t)} \leq K_{1}
$$

whenever $x \in \mathbf{R}$ and $t>0$. By (7.7), this is seen to be true provided that (7.12) holds whenever $x, x \pm t$ belong to some fixed interval of length $2 / N$ (cf. [3, subsection 3.5]).

7.7. We make the preliminary observation that

$$
0<\varepsilon_{3} \leq N \theta_{1}=\theta_{1} /(1 / N) \leq 1-\varepsilon_{3}<1 .
$$

If $N=2 k+1 \geq 3$, set $\beta=c^{k}$ so that $h(\beta)=\bar{c}^{k}=\bar{\beta}=c^{k+1}$ and $h(\bar{\beta})=\beta$. For the quadruple $Z=\left(1, z_{1}, c, \bar{\beta}\right)$, we have $h Z=\left(1, \bar{c} z_{1}, \bar{c}, \beta\right)$. If $\theta_{1} \leq$ $(2 N)^{-1}$ then

$$
C(h Z)=\frac{\beta-1}{\beta-\bar{c} z_{1}} \frac{\bar{c}-\bar{c} z_{1}}{\bar{c}-1}=\frac{\beta-1}{\beta-\bar{c} z_{1}} \frac{1-z_{1}}{1-c}
$$

satisfies

$$
C(h Z) \leq \frac{2}{|e(1 / 3)-1|} \frac{2 \sin \left(\pi \theta_{1}\right)}{2 \sin (\pi / N)} \leq \frac{2 \pi \theta_{1}}{\sqrt{3}(2 / \pi)(\pi / N)}=\frac{\pi N \theta_{1}}{\sqrt{3}} \leq \frac{\pi}{2 \sqrt{3}}<0.91
$$

so that $C(Z) \leq 1-\varepsilon_{4}$ since $h^{-1}=h$ is $K$-qs. Thus

$$
\begin{aligned}
\varepsilon_{4} & \leq 1-C(Z)=C\left(\bar{\beta}, 1, z_{1}, c\right)=\left|\frac{c-\bar{\beta}}{c-1} \frac{z_{1}-1}{z_{1}-\bar{\beta}}\right| \\
& \leq \frac{2}{\sqrt{3}} \frac{2 \sin \left(\pi \theta_{1}\right)}{2 \sin (\pi / N)} \leq \frac{\pi}{\sqrt{3}} N \theta_{1},
\end{aligned}
$$

which gives (7.13). If $(2 N)^{-1}<\theta_{1}<N^{-1}$, we see in the same way that $C(Z) \leq A<1$ where $A$ is an absolute constant, and thus $C(h Z) \leq 1-\varepsilon_{4}$. This, in turn, implies that $\left|z_{1}-c\right| \geq \varepsilon_{5}|c-1|$, so that (7.13) holds. If $N=2 k \geq 4$, we argue in the same way, with $\beta=\bar{\beta}=h(\beta)=c^{k}=-1$.

If $N=2$ so that $h$ fixes the points \pm 1 , we consider the quadruple $Z=$ $\left(1, z_{1},-1,-i\right)$. Now $c=-1$ and $h\left(z_{1}\right)=-z_{1}$. Suppose first that $\theta_{1} \leq$ $(2 N)^{-1}=\frac{1}{4}$ so that $z_{1} \in(1, i]$. Then

$$
\begin{aligned}
1-C(Z) & =C\left(z_{1},-1,-i, 1\right)=\frac{1}{\sqrt{2}} \frac{\sin \left(\pi \theta_{1}\right)}{\sin \left[\pi\left(1+2 \theta_{1}\right) / 4\right]} \\
& \leq \frac{\pi \theta_{1} \sqrt{2}}{1+2 \theta_{1}} \leq \frac{\pi \sqrt{2}}{6}<\frac{3}{4} .
\end{aligned}
$$


Thus $C(Z)>\frac{1}{4}$ and so

$$
\varepsilon_{6} \leq C(h Z)=C\left(1,-z_{1},-1, h(-i)\right)=\left|\frac{1-z_{1}}{2}\right|\left|\frac{h(-i)-1}{h(-i)+z_{1}}\right| .
$$

Since $h$ is sense-reversing and $i \in\left[z_{1},-1\right)$, the point $h(i)$ lies on the arc $\left(-1, h\left(z_{1}\right)\right]$, so that $h(-i)=-h(i) \in\left(1, h^{2}\left(z_{1}\right)\right]=\left(1, z_{1}\right]$. Note that $h(-z)=$ $-h(z)$ by (7.2) since $c=-1$. It follows that

$$
\left|\frac{h(-i)-1}{h(-i)+z_{1}}\right| \leq\left|\frac{1-z_{1}}{2 z_{1}}\right|=\frac{\left|1-z_{1}\right|}{2} \text {. }
$$

Hence $\left|1-z_{1}\right|=2 \sin \left(\pi \theta_{1}\right) \geq 2 \sqrt{\varepsilon_{6}}$ and (7.13) follows, with $N=2$. If $\frac{1}{4}<\theta_{1}<\frac{1}{2}$, we argue in the same way. This completes the proof of (7.13).

7.8. We continue with the proof of (7.12). By the definition of $f$, we have

$$
f_{0}(x)=x /\left(2 N \theta_{1}\right) \text { for } 0 \leq x \leq \theta_{1}
$$

and

$$
f_{0}(x)=-f_{0}\left(h_{0}(x)\right)=-h_{0}(x) /\left(2 N \theta_{1}\right) \text { for } \theta_{1}-N^{-1} \leq x<0 .
$$

Suppose that $h_{0}$ is $K_{1}$-qs on $\left[\theta_{1}-N^{-1}, 0\right]$. Recall that we say that $h_{0}$ is $K_{1}$-qs on an interval $I$ if and only if

$$
\frac{1}{K_{1}} \leq \frac{h_{0}(x+t)-h_{0}(x)}{h_{0}(x)-h_{0}(x-t)} \leq K_{1}
$$

whenever $t>0$ and $x, x \pm t \in I$. If, furthermore,

$$
K_{1}^{-1} \leq \frac{h_{0}(-x)}{x}=-\frac{f_{0}(-x)}{f_{0}(x)} \leq K_{1} \text { for } 0<x \leq \min \left\{\theta_{1}, N^{-1}-\theta_{1}\right\},
$$

we may apply [2, Lemma 4 , p. 322] to $f_{0}$ on the intervals $\left[\theta_{1}-N^{-1}, 0\right]$ and $\left[0, \theta_{1}\right]$ to conclude that $f_{0}$ is $K_{1}$-qs on $\left[\theta_{1}-N^{-1}, \theta_{1}\right]$.

By (7.7), it follows that $f_{0}$ is $K_{1}$-qs on $\left[\theta_{1}, \theta_{1}+N^{-1}\right]$ also and thus $K_{1}$-qs on $\left[\theta_{1}-N^{-1}, \theta_{1}+N^{-1}\right]$, by [3, Lemma A, p. 8] and by (7.13), provided that

$$
\frac{1}{K_{1}} \leq \frac{f_{0}\left(\theta_{1}+t\right)-f_{0}\left(\theta_{1}\right)}{f_{0}\left(\theta_{1}\right)-f_{0}\left(\theta_{1}-t\right)} \leq K_{1}
$$

for $0<t \leq \theta_{2}=\min \left\{\theta_{1}, N^{-1}-\theta_{1}\right\}$. Note that [3, Lemma A] is a slight extension of [2, Lemma 4]. Since $\theta_{2} \geq \varepsilon_{3} N^{-1}$ by (7.13), it suffices to prove (7.18) for $0<t \leq \theta_{2}$ rather than for $0<t \leq N^{-1}$, that is, whenever $\theta_{1}$ and $\theta_{1} \pm t$ belong to $\left[\theta_{1}-N^{-1}, \theta_{1}+N^{-1}\right]$. Thus to complete the proof that $f_{0}$ is $K_{1}$-qs on $\overline{\mathbf{R}}$, it remains to establish (7.16)-(7.18), which we now proceed to do.

7.9. To prove (7.16), suppose that $x_{1}<x_{2}<x_{3}<x_{4}$ and that either $x_{i} \in$ $\left[-N^{-1}, 0\right]$ for $1 \leq i \leq 4$ or $x_{i} \in\left[\theta_{1}-N^{-1}, \theta_{1}\right]$, for $1 \leq i \leq 4$. Suppose further that

$$
0<\varepsilon_{7} \leq C\left(x_{1}, x_{2}, x_{3}, x_{4}\right) \leq 1-\varepsilon_{7},
$$


for some $\varepsilon_{7}=\varepsilon_{7}(K)$ to be determined later. Set $z_{j}=e\left(x_{j}\right)$ for $1 \leq j \leq 4$. Then $z_{1}, z_{2}, z_{3}$, and $z_{4}$ follow each other in the positive direction on an arc of $\mathbf{T}$ of length not exceeding $2 \pi / N \leq \pi$, and so, by (7.2) or (7.8), the $h\left(z_{i}\right)$ follow each other in the negative direction on an arc of length not exceeding $2 \pi / N$. Thus

$$
0<\varepsilon_{8} \leq C\left(z_{1}, z_{2}, z_{3}, z_{4}\right) \leq 1-\varepsilon_{8}<1
$$

and so

$$
0<\varepsilon_{9} \leq C\left(h\left(z_{1}\right), h\left(z_{2}\right), h\left(z_{3}\right), h\left(z_{4}\right)\right) \leq 1-\varepsilon_{9}<1
$$

Therefore

$$
0<\varepsilon_{10} \leq C\left(h_{0}\left(x_{1}\right), h_{0}\left(x_{2}\right), h_{0}\left(x_{3}\right), h_{0}\left(x_{4}\right)\right) \leq 1-\varepsilon_{10}<1 .
$$

Now (7.20) implies (7.16) as follows. Define $I_{1}=\left[-N^{-1}, 0\right]$ and $I_{2}=$ $\left[0, \theta_{1}\right]$. If $x, x \pm t \in I_{1}$, we apply (7.19) to

$$
x_{1}=x-t, \quad x_{2}=x, \quad x_{3}=x+t, \quad \text { and } x_{4}=\theta_{1} .
$$

Then

$$
\frac{1}{2} \leq C\left(x_{1}, x_{2}, x_{3}, x_{4}\right)=\frac{1}{2}\left\{1+t /\left(\theta_{1}-x\right)\right\}=\gamma,
$$

say. If $-N^{-1} / 2 \leq x<0$, then $t \leq-x$ and

$$
\gamma \leq \frac{1}{2}\left\{1-\frac{x}{\theta_{1}-x}\right\} \leq 1-\frac{N \theta_{1}}{2\left(1+N \theta_{1}\right)} \leq 1-\frac{\varepsilon_{3}}{2\left(1+\varepsilon_{3}\right)}<1
$$

by (7.13). If $-N^{-1}<x<-N^{-1} / 2$, then $t \leq N^{-1}+x$ and

$$
\gamma \leq 1-N \theta_{1}\left(2 N \theta_{1}+1\right)^{-1} \leq 1-\varepsilon_{3}\left(1+2 \varepsilon_{3}\right)^{-1} .
$$

Thus (7.19) holds with $\varepsilon_{7}=\varepsilon_{3}\left(2 \varepsilon_{3}+2\right)^{-1}$. Therefore (7.20) is also valid, and consequently

$$
\varepsilon_{10} \leq \frac{h_{0}(x+t)-h_{0}(x)}{h_{0}(x+t)-h_{0}(x-t)} \frac{h_{0}\left(\theta_{1}\right)-h_{0}(x-t)}{h_{0}\left(\theta_{1}\right)-h_{0}(x)} .
$$

Now $h_{0}(x) \geq 0$ and $h_{0}(x \pm t) \geq 0$ while

$$
h_{0}\left(\theta_{1}\right)=\theta_{1}-N^{-1}=-N^{-1}\left(1-N \theta_{1}\right) \leq-\varepsilon_{3} N^{-1}
$$

by (7.9) and (7.13). Since also $h_{0}(x-t) \leq h_{0}\left(-N^{-1}\right)=N^{-1}$, we obtain

$$
\frac{h_{0}(x+t)-h_{0}(x-t)}{h_{0}(x+t)-h_{0}(x)} \varepsilon_{10} \leq \frac{-\varepsilon_{3} N^{-1}-N^{-1}}{-\varepsilon_{3} N^{-1}}=\frac{\varepsilon_{3}+1}{\varepsilon_{3}} \leq \frac{2}{\varepsilon_{3}},
$$

which gives the left-hand inequality (7.16).

By (7.20) and (7.6) we have, furthermore,

$$
\begin{aligned}
\varepsilon_{10} & \leq C\left(h_{0}\left(x_{4}\right), h_{0}\left(x_{1}\right), h_{0}\left(x_{2}\right), h_{0}\left(x_{3}\right)\right) \\
& =\frac{h_{0}(x)-h_{0}(x-t)}{h_{0}(x+t)-h_{0}(x-t)} \frac{h_{0}\left(\theta_{1}\right)-h_{0}(x+t)}{h_{0}\left(\theta_{1}\right)-h_{0}(x)} \leq \frac{h_{0}(x)-h_{0}(x-t)}{h_{0}(x+t)-h_{0}(x-t)} .
\end{aligned}
$$


This yields the right-hand inequality (7.16), and we have proved (7.16) whenever $x, x \pm t \in I_{1}$.

If $x, x \pm t \in I_{2}$, we see in the same way that (7.16) holds, by considering the quadruple

$$
x_{1}=\theta_{1}-N^{-1}, \quad x_{2}=x-t, \quad x_{3}=x, \quad \text { and } x_{4}=x+t .
$$

Suppose that $0<t \leq \frac{1}{2} \min \left\{\theta_{1}, N^{-1}-\theta_{1}\right\}$. Then we see that (7.16) holds with $x=0$, by considering the quadruple $x_{1}=-t, x_{2}=0, x_{3}=t$, and $x_{4}=\theta_{1}$, and by noting that

$$
\frac{h_{0}\left(\theta_{1}\right)-h_{0}(-t)}{h_{0}\left(\theta_{1}\right)} \leq \frac{2 N^{-1}-\theta_{1}}{N^{-1}-\theta_{1}} \leq \frac{2}{\varepsilon_{3}},
$$

say, by (7.9) and (7.13).

Now [3, Lemma A] implies that (7.16) holds whenever $x, x \pm t \in I_{1} \cup I_{2}=$ $\left[-N^{-1}, \theta_{1}\right]$. This completes the proof of (7.16).

7.10. We proved (7.16) on the larger interval $\left[-N^{-1}, \theta_{1}\right]$ in order to use it to establish (7.17) and (7.18). Since $h_{0}(0)=0$, we get from (7.16) that

$$
K_{1}^{-1} \leq\left|h_{0}(x) / h_{0}(-x)\right| \leq K_{1}
$$

for $0<x \leq \min \left\{\theta_{1}, N^{-1}-\theta_{1}\right\}$. Next note that since $e\left(h_{0}(\theta)\right)=h(e(\theta))$ and $h^{2}=\mathrm{Id}$, we have $e\left(h_{0}^{2}(\theta)\right)=e(\theta)$ for all real $\theta$. Thus $h_{0}^{2}(x)=x+n$ for some integer $n$, for all real $x$. Since $h_{0}(0)=0$ so that $h_{0}^{2}(0)=0$, we have

$$
h_{0}^{2}=\mathrm{Id} \text {. }
$$

Now we obtain (7.17) by reasoning as in [3, subsection 3.4]. Thus, for example, if $0<x<h_{0}(-x)$, then $0>h_{0}(x)>h_{0}^{2}(-x)=-x$ so that $0<-h_{0}(x) / x<1$. By $(7.21)$, we have

$$
1<\frac{h_{0}(-x)}{x}=\frac{h_{0}(-x)}{-h_{0}(x)} \frac{-h_{0}(x)}{x}<K_{1},
$$

and (7.17) holds. If $0<h_{0}(-x) \leq x$, we argue in the same way. This proves (7.17).

7.11. It remains to prove (7.18). By (7.14) and (7.13), we have $f_{0}\left(\theta_{1}\right)$ $f_{0}\left(\theta_{1}-t\right)=t /\left(2 N \theta_{1}\right)$ for $0<t \leq \theta_{2}$. By (7.7), (7.15), and (7.8), we obtain

$$
\begin{aligned}
f_{0}\left(\theta_{1}+t\right)-f_{0}\left(\theta_{1}\right) & =f_{0}\left(\theta_{1}-N^{-1}+t\right)-f_{0}\left(\theta_{1}-N^{-1}\right) \\
& =\frac{h_{0}\left(\theta_{1}-N^{-1}\right)-h_{0}\left(\theta_{1}-N^{-1}+t\right)}{2 N \theta_{1}}=\frac{h_{0}\left(\theta_{1}\right)-h_{0}\left(\theta_{1}+t\right)}{2 N \theta_{1}}
\end{aligned}
$$

for $0<t \leq \theta_{2}$. Thus we need to show that

$$
K_{1}^{-1} \leq\left[h_{0}\left(\theta_{1}\right)-h_{0}\left(\theta_{1}+t\right)\right] / t \leq K_{1} \quad \text { for } 0<t \leq \theta_{2} .
$$


The proof of (7.23) is similar to the estimation of the quantity in [3, (3.16), p. 16]. Suppose first that $\delta \equiv h_{0}\left(\theta_{1}\right)-h_{0}\left(\theta_{1}+t\right) \geq t$. We have $h_{0}\left(\theta_{1}+t\right)=$ $h_{0}\left(\theta_{1}\right)-\delta=\theta_{1}-N^{-1}-\delta$. By this, (7.22), and (7.9), we get $h_{0}\left(\theta_{1}-N^{-1}-\delta\right)=$ $h_{0}^{2}\left(\theta_{1}+t\right)=\theta_{1}+t=h_{0}\left(\theta_{1}-N^{-1}\right)+t$. Thus $h_{0}\left(\theta_{1}-N^{-1}-\delta\right)-h_{0}\left(\theta_{1}-N^{-1}\right)=t$. We proved (7.16) when $x, x \pm t \in\left[-N^{-1}, 0\right]$. Thus $h_{0}$ is $K_{1}$-qs on $\left[-N^{-1}, 0\right]$, which contains the interval $\left[\theta_{1}-N^{-1}-\theta_{2}, \theta_{1}-N^{-1}+\theta_{2}\right]$, and consequently

$$
\begin{gathered}
h_{0}\left(\theta_{1}-N^{-1}\right)-h_{0}\left(\theta_{1}-N^{-1}+t\right) \leq K_{1}\left\{h_{0}\left(\theta_{1}-N^{-1}-t\right)-h_{0}\left(\theta_{1}-N^{-1}\right)\right\} \\
\leq K_{1}\left\{h_{0}\left(\theta_{1}-N^{-1}-\delta\right)-h_{0}\left(\theta_{1}-N^{-1}\right)\right\}=K_{1} t
\end{gathered}
$$

since $h_{0}$ is decreasing and $\delta \geq t$. So by(7.8),

$$
\delta=h_{0}\left(\theta_{1}-N^{-1}\right)-h_{0}\left(\theta_{1}-N^{-1}+t\right) \leq K_{1} t,
$$

and (7.23) holds. If $\delta<t$, we get similarly

$$
\begin{aligned}
t & =h_{0}\left(\theta_{1}-N^{-1}-\delta\right)-h_{0}\left(\theta_{1}-N^{-1}\right) \leq h_{0}\left(\theta_{1}-N^{-1}-t\right)-h_{0}\left(\theta_{1}-N^{-1}\right) \\
& \leq K_{1}\left\{h_{0}\left(\theta_{1}-N^{-1}\right)-h_{0}\left(\theta_{1}-N^{-1}+t\right)\right\}=K_{1} \delta,
\end{aligned}
$$

which implies (7.23). This completes the proof of (7.23) and hence that of (7.18). Thus the proof of (7.5) is also complete.

Theorem 2 is proved.

\section{NONDISCRETE GROUPS}

8.1. We shall prove the following result, after which case (iv) of Theorem 1 can be handled essentially by quoting arguments from [3].

Lemma 14. Let $G$ be a closed nondiscrete convergence group. Then there is a homeomorphism $f$ of $\mathbf{T}$ onto itself such that $f \circ G \circ f^{-1}$ contains $\mathscr{R}$, or there is a homeomorphism $f$ of $\mathbf{T}$ onto $\overline{\mathbf{R}}$ such that $f \circ G \circ f^{-1}$ contains $\mathscr{T}$ or $\mathscr{M}$.

Suppose that $G$ is a closed nondiscrete convergence group and that Lemma 14 holds. By performing a preliminary conjugation, we may assume that $G$ contains $\mathscr{T}, \mathscr{M}$, or $\mathscr{R}$. If $G$ contains $\mathscr{T}$ or $\mathscr{R}$, we may proceed as in the proof of [3, Lemma 10] and show that $G$ is a Möbius group. If $\mathscr{M} \subset G$ we may prove as in that lemma that there is a homeomorphism $f$ of the form

$$
f(x)=A x^{r} \text { for } 0 \leq x \leq \infty
$$

and

$$
f(x)=-B|x|^{r} \text { for }-\infty<x<0,
$$

where $A, B$, and $r$ are positive numbers, such that $f^{-1} \circ G \circ f$ is a Möbius group. In view of Lemmas 4 and 5, this then proves Theorem 1 in case (iv). As the proof of [3, Lemma 10] works for convergence groups instead of uniformly quasisymmetric groups without any essential changes, we do not give any further details here. 
Lemma 14 corresponds to the results given in [3, §9] for nondiscrete uniformly quasisymmetric groups, some of which are contained in [3, Lemma 9]. The proof of Lemma 14 must be essentially different from the arguments given in [3] since the conjugating homeomorphisms for cyclic subgroups of $G$ are, again, not known to form a normal family.

Let $G$ be nondiscrete and closed. As in [3, subsection 9.1] and in view of Lemma 4, we can find a sequence $g_{n}$ of distinct sense-preserving elements of $G$ tending to Id uniformly on $\mathbf{T}$ (with $g_{n}^{-1} \rightarrow$ Id also) as $n \rightarrow \infty$, such that each $g_{n}$ can be conjugated to the same type of Möbius transformation: parabolic, hyperbolic, or elliptic. We shall use these terms of the $g_{n}$ also.

Recall the notation (2.1), which will be used repeatedly.

8.2. Suppose that each $g_{n}$ is parabolic with fixed point $\alpha_{n}$. By passing to a subsequence, we may assume that $\alpha_{n} \rightarrow \alpha \in \mathbf{T}$. We choose $\beta \in \mathbf{T}, \beta \neq \alpha$, and show that for each $\gamma \in \mathbf{T}$ with $\alpha \neq \gamma \neq \beta$, there is a parabolic element $g$ of $G$ fixing $\alpha$ such that $g(\beta)=\gamma$.

Let $\beta$ and $\gamma$ be given, and let $I_{n}$ be an arc containing $\gamma$ but not $\alpha$ or $\beta$, with $\left|I_{n}\right|<1 / n$. Since $g_{n} \rightarrow$ Id uniformly on $\mathbf{T}$, we can find, for all sufficiently large $n$, an integer $m(n)$ such that $h_{n}(\beta) \in I_{n}$ where $h_{n}=g_{n}^{m(n)} \in G$. Since $G$ is a closed convergence group, we may pass to a subsequence and assume that either $h_{n} \rightarrow g \in G$ uniformly on $\mathbf{T}$, or $h_{n} \rightarrow x_{0}$ and $h_{n}^{-1} \rightarrow y_{0}$ uniformly on compact subsets of $\mathbf{T} \backslash\left\{y_{0}\right\}$ and $\mathbf{T} \backslash\left\{x_{0}\right\}$, respectively, for some $x_{0}, y_{0} \in \mathbf{T}$.

To eliminate the latter possibility, suppose that it holds. Write $\gamma_{n}=h_{n}(\beta) \rightarrow$ $\gamma$, and let $Q_{n}$ be the Möbius transformation of $\mathbf{T}$ onto itself with $Q_{n}(\alpha)=\alpha_{n}$, $Q_{n}(\beta)=\beta$, and $Q_{n}(\gamma)=\gamma_{n}$. Then $Q_{n} \rightarrow$ Id uniformly on $\mathbf{T}$ as $n \rightarrow \infty$, and $\lambda_{n}=Q_{n}^{-1} \circ h_{n} \circ Q_{n} \rightarrow x_{0}$ while $\lambda_{n}^{-1} \rightarrow y_{0}$ uniformly on compact subsets of $\mathbf{T} \backslash\left\{y_{0}\right\}$ and $\mathbf{T} \backslash\left\{x_{0}\right\}$, respectively. On the other hand, we have $\lambda_{n}(\alpha)=\alpha$, $\lambda_{n}(\beta)=\gamma$, and $\lambda_{n}^{-1}(\gamma)=\beta$ for all $n$, and at least one of $\alpha, \beta$, and $\gamma$ is not among $x_{0}$ and $y_{0}$. Considering all possibilities, we see that either $x_{0}=\gamma$, $y_{0}=\alpha$ or $x_{0}=\alpha, y_{0}=\beta$.

Without loss of generality, we assume that $x_{0}=\gamma$ and $y_{0}=\alpha$, that the points $\alpha, \beta$, and $\gamma$ follow each other in the positive direction on the circle, and that either $\alpha_{n} \in[\alpha, c \alpha]$ for all $n$ or $\alpha_{n} \in[\bar{c} \alpha, \alpha]$ for all $n$ where $c=e(\varepsilon)$ and $\varepsilon$ is a sufficiently small but fixed positive number such that $\beta, \gamma \notin[\bar{c} \alpha, c \alpha]=J$, say. Now consider the fact that $h_{n}$ is parabolic with fixed point $\alpha_{n}$ and that $h_{n}$ moves $\beta$ in the direction of $\gamma$. If $\alpha_{n} \in[\alpha, c \alpha]$ then $h_{n}(\bar{c} \alpha) \in J$. If $\alpha_{n} \in[\bar{c} \alpha, \alpha]$ then $h_{n}(\bar{c} \alpha) \in[\bar{c} \alpha, \alpha]$. Hence $h_{n}(\bar{c} \alpha)$ does not tend to $\gamma$, which is a contradiction. We conclude that $h_{n} \rightarrow g \in G$ uniformly on $\mathbf{T}$.

Clearly $g(\alpha)=\alpha$ and $g(\beta)=\gamma$, so that $g \neq \mathrm{Id}$. Also $g$ is sense-preserving but not elliptic. If $g$ is not parabolic, then $g$ is hyperbolic and $g\left(z_{0}\right)=z_{0}$ for some $z_{0}$ distinct from $\alpha, \beta$, and $\gamma$. We may again assume that $\beta \in(\alpha, \gamma)$. Clearly $z_{0} \in(\alpha, \beta)$ or $z_{0} \in(\gamma, \alpha)$. If $z_{0} \in(\gamma, \alpha)$ then, since $g$ moves $\beta$ toward $z_{0}$, the point $z_{0}$ must be the attractive fixed point of $g$. Hence, if 
$z_{1} \in\left(z_{0}, \alpha\right)$, we have $g\left(z_{1}\right) \in\left(z_{0}, z_{1}\right)$. But $h_{n}\left(z_{1}\right) \in\left(z_{1}, \alpha_{n}\right)$ for all $n$ and so $g\left(z_{1}\right) \in\left[z_{1}, \alpha\right]$, which is a contradiction. If $z_{0} \in(\alpha, \beta)$, we argue in the same way. Hence $g$ is parabolic.

Using Lemma 10, we can now find a homeomorphism $f: \mathbf{T} \rightarrow \overline{\mathbf{R}}$ with $f(\alpha)=$ $\infty$ such that $f \circ G \circ f^{-1}$ contains $\mathscr{T}$.

8.3. Suppose that each $g_{n}$ is hyperbolic with fixed points $\alpha_{n}$ and $\beta_{n}$, and that $\alpha_{n} \rightarrow \alpha$ and $\beta_{n} \rightarrow \beta$ as $n \rightarrow \infty$. If $\alpha \neq \beta$, let $I$ be one of the open arcs joining $\alpha$ and $\beta$, and choose $\gamma \in I$. Proceeding as in Subsection 8.2, we can show that for each $\delta \in I \backslash\{\gamma\}$ there is a hyperbolic element $g$ of $G$ fixing $\alpha$ and $\beta$ such that $g(\gamma)=\delta$. By using Lemma 10 we find a homeomorphism $f$ of $\mathbf{T}$ onto $\overline{\mathbf{R}}$ with $f(\alpha)=0$ and $f(\beta)=\infty$ such that $f \circ G \circ f^{-1}$ contains $\mathscr{M}$.

If $\alpha=\beta$, we choose $\gamma \neq \alpha$ and show that for each $\delta \in \mathbf{T} \backslash\{\alpha, \gamma\}$ there is a parabolic element $g$ of $G$ fixing $\alpha$ such that $g(\gamma)=\delta$. Then it will follow as in Subsection 8.2 that there is a homeomorphism $f$ such that $f \circ G \circ f^{-1}$ contains $\mathscr{T}$. As before, we choose $h_{n}=g_{n}^{m(n)}$ so that $\left|\delta_{n}-\delta\right|<1 / n$ where $\delta_{n}=h_{n}(\gamma)$. By using the methods of Subsection 8.2 and by passing to a subsequence, if necessary, we see that $h_{n} \rightarrow g \in G$ uniformly on $\mathbf{T}$ as $n \rightarrow \infty$, where $g$ is parabolic with $g(\alpha)=\alpha$ and $g(\gamma)=\delta$, as required.

8.4. Suppose that each $g_{n}$ is elliptic and conjugate to $R_{\alpha(n)}$ where $0<|\alpha(n)| \leq$ $\frac{1}{2}$. By passing to a subsequence and replacing $g_{n}$ by $g_{n}^{-1}$, if necessary, we may assume that $\alpha(n)>0$ for all $n$. As in [3, Subsection 9.2], we see that $\alpha(n) \rightarrow 0$ as $n \rightarrow \infty$.

If there are $g \in G$ and a homeomorphism $f$ such that $f \circ g \circ f^{-1}=R_{\alpha}$ where $\alpha$ is irrational, then $G_{1}=f \circ G \circ f^{-1}$ contains $R_{n \alpha}$ for all integers $n$. Since $G$ and hence $G_{1}$ is closed, we have $\mathscr{R} \subset G_{1}$. So we may assume from now on that all elliptic elements of $G$ are of finite order.

8.4.1. It is convenient to formulate a few auxiliary results as lemmas.

Lemma 15. Suppose that $h_{n}=g_{n}^{m(n)} \rightarrow h$ as $n \rightarrow \infty$ uniformly on $\mathbf{T}$ where $h$ is a homeomorphism and each $m(n)$ is a positive integer. Then $h \in G$, and if $h \neq \mathrm{Id}$, then $h$ is elliptic or parabolic. If $h \neq \operatorname{Id}$ then $m(n) \rightarrow \infty$ as $n \rightarrow \infty$.

Proof. Since $g_{n} \rightarrow \mathrm{Id}$, we have $g_{n}^{k} \rightarrow \mathrm{Id}$ for any fixed $k$. Thus $m(n) \rightarrow \infty$ if $h \neq \mathrm{Id}$. We have $h \in G$ since $G$ is closed, and clearly $h$ is sense-preserving. If $h \neq \mathrm{Id}$ and if $h$ is not elliptic or parabolic, then $h$ is hyperbolic. Suppose that $h$ has attractive fixed point $z_{1}$ and repulsive fixed point $z_{2}$, and pick $z_{3} \in\left(z_{1}, z_{2}\right)$. Then $h\left(z_{3}\right) \in\left(z_{1}, z_{3}\right)$. Thus $h_{n}\left(z_{3}\right) \in\left(z_{1}, z_{3}\right)$ for all large $n$. Hence for any $z_{4} \in\left(z_{2}, z_{1}\right)$, we have $h_{n}\left(z_{4}\right) \in\left(z_{2}, z_{4}\right)$ for all large $n$, for if not, then $h_{n}$ will have a fixed point on $\left[z_{3}, z_{4}\right]$ for topological reasons. It follows that $h\left(z_{4}\right) \in\left[z_{2}, z_{4}\right]$, which is a contradiction since $z_{1}$ is the attractive fixed point of $h$. This proves Lemma 15 . 
Note that a function $g_{n}^{m(n)}$ is either elliptic or the identity. If $g_{n}^{m(n)}$ does not tend to Id, then $g_{n}^{m(n)}$ is elliptic for all large $n$.

8.4.2. Lemma 16. If the $m(n)$ are positive integers and if $g_{n}^{m(n)} \rightarrow x_{0}$ and $g_{n}^{-m(n)} \rightarrow y_{0}$ as $n \rightarrow \infty$, locally uniformly on $\mathbf{T} \backslash\left\{y_{0}\right\}$ and $\mathbf{T} \backslash\left\{x_{0}\right\}$, respectively, then $x_{0}=y_{0}$. Furthermore, $m(n) \rightarrow \infty$ as $n \rightarrow \infty$.

Proof. We see that $m(n) \rightarrow \infty$ as in the proof of Lemma 15. Suppose that $x_{0} \neq y_{0}$. Then, if the positive number $\varepsilon$ is small enough and $n$ is large enough, the function $g_{n}^{m(n)}$ maps $\left[c y_{0}, \bar{c} y_{0}\right]$ into $\left(\bar{c} x_{0}, c x_{0}\right)$ where $c=e(\varepsilon)$. Hence the image of $J=\left[\bar{c} y_{0}, c y_{0}\right]$ under $g_{n}^{m(n)}$ contains $\left[c x_{0}, \bar{c} x_{0}\right]$, which, in turn, contains $J$ is $\varepsilon$ is small enough. This implies, for topological reasons, that $g_{n}^{m(n)}$ has a fixed point on $J$, which is a contradiction. This proves Lemma 16.

Lemma 17. If $g_{n}^{2 m(n)} \rightarrow h \in G$ where $h$ is parabolic, and if $g_{n}^{m(n)} \rightarrow x_{0}$ and $g_{n}^{-m(n)} \rightarrow y_{0}$ uniformly on compact subsets of $\mathbf{T} \backslash\left\{y_{0}\right\}$ and $\mathbf{T} \backslash\left\{x_{0}\right\}$, respectively, then $x_{0}=y_{0}$ and $x_{0}$ is the fixed point of $h$.

Proof. By Lemma 16, we have $x_{0}=y_{0}$. We claim that $h\left(x_{0}\right)=x_{0}$. If $I$ is any open arc containing $x_{0}$ and if $J=\mathbf{T} \backslash I$, then $g_{n}^{m(n)}(J) \subset I$ and so $g_{n}^{m(n)}(I) \supset J$ for all large $n$, since $g_{n}^{m(n)}$ is a homeomorphism of $\mathbf{T}$. Thus $g_{n}^{2 m(n)}(I) \supset g_{n}^{m(n)}(J)=J_{n}$, say, and $\left|J_{n}\right| \rightarrow 0, J_{n} \rightarrow x_{0}$. It follows that $x_{0} \in$ $\overline{h(I)}$. Since $I$ was an arbitrary interval containing $x_{0}$, we have $h\left(x_{0}\right)=x_{0}$, as asserted. This proves Lemma 17.

8.4.3. Lemma 18. Suppose that $a$ and $b$ are distinct points of $\mathbf{T}$, that $0<$ $m(n) \alpha(n)<1$, and that $g_{n}^{m(n)} \rightarrow g \in G$ where $g(a)=b$. Then $g$ is elliptic or $g$ is parabolic with fixed point $z_{0}$ distinct from $a$ and $b$. Further, there is a sequence $k(n)$ of integers with $|k(n)| \rightarrow \infty$ such that $g_{n}^{k(n)} \rightarrow h \in G$ where $h^{2}=g$. The function $h$ is parabolic if and only if $g$ is, and then $h\left(z_{0}\right)=g\left(z_{0}\right)=z_{0}$.

Proof. Since $g_{n} \rightarrow \mathrm{Id}$, we may assume that each $m(n)$ is even and write $m(n)=2 p(n)$. If a subsequence of $g_{n}^{p(n)}$ tends to a homeomorphism $h$, then $h \in G$ since $G$ is closed, and the remaining statements about $h$ are obvious. Note that $g \neq$ Id since $g(a)=b \neq a$, so that $g$ is elliptic or parabolic by Lemma 15.

If no subsequence of $g_{n}^{p(n)}$ tends to a homeomorphism, then $g_{n}^{p(n)} \rightarrow x_{0}$, and $g_{n}^{-p(n)} \rightarrow x_{0}$ also, by Lemma 16 (we retain the notation $p(n)$ for any subsequence that we consider). If $x_{1} \notin\left\{x_{0}, g^{-1}\left(x_{0}\right)\right\}$, then

$$
g_{n}^{3 p(n)}\left(x_{1}\right)=g_{n}^{2 p(n)}\left(g_{n}^{p(n)}\left(x_{1}\right)\right) \rightarrow g\left(x_{0}\right)
$$

and

$$
g_{n}^{3 p(n)}\left(x_{1}\right)=g_{n}^{p(n)}\left(g_{n}^{2 p(n)}\left(x_{1}\right)\right) \rightarrow x_{0}
$$


since $g_{n}^{2 p(n)}\left(x_{1}\right) \rightarrow g\left(x_{1}\right) \neq x_{0}$. Hence $g\left(x_{0}\right)=x_{0}$ so that $g$ is parabolic and $z_{0}=x_{0}$. Thus $x_{0} \neq a, b$.

By Lemma 4, there is a homeomorphism $f_{n}$ such that $f_{n}^{-1} \circ g_{n} \circ f_{n}=R_{\alpha(n)}$. Since $\alpha(n)>0$ and $0<2 p(n) \alpha(n)<1$, the points $a, g_{n}(a), g_{n}^{2}(a), \ldots$, $g_{n}^{2 p(n)}(a)$, and $a$ follow each other in the positive direction on $\mathbf{T}$. Hence, in particular,

$$
g_{n}^{p(n)}(a) \in\left(a, g_{n}^{2 p(n)}(a)\right) \subset(a, c b)
$$

for all large $n$. Here and later, $c=e(\varepsilon)$ and $\varepsilon$ is any small positive number. Thus $x_{0} \in(a, b)$ since $g_{n}^{p(n)}(a) \rightarrow x_{0}$.

We may assume that $g_{n}^{-p(n)}\left(x_{0}\right) \rightarrow x_{1}$ and $g_{n}^{p(n)}\left(x_{0}\right) \rightarrow x_{2}$, so that $g\left(x_{1}\right)=$ $x_{2}$. If $x_{1}=x_{2}=x_{0}$ then for all large $n$, we have $g_{n}^{-p(n)}\left(x_{0}\right) \in\left(a, x_{0}\right)$ and $g_{n}^{p(n)}\left(x_{0}\right) \in\left(x_{0}, \bar{c} b\right)$, so that $g_{n}^{2 p(n)}(a) \in(a, \bar{c} b)$. Since $g_{n}^{2 p(n)}(a) \rightarrow b$, this is impossible. Thus $x_{1} \neq x_{2}$. Since $0<2 p(n) \alpha(n)<1$, we have $g_{n}^{p(n)}\left(x_{0}\right) \in$ $\left(x_{0}, g_{n}^{-p(n)}\left(x_{0}\right)\right)$ for all $n$. Hence $x_{0} \in\left(x_{1}, x_{2}\right)$ and $x_{0} \notin\left[x_{2}, x_{1}\right]$.

Since $g_{n} \rightarrow$ Id, we can find a sequence $k(n) \rightarrow \infty$ such that $g_{n}^{2 k(n)}\left(x_{2}\right) \rightarrow$ $x_{1}$. We have $2 k(n) \leq p(n)$ for all large $n$. For if $2 k(n)>p(n)$ for some subsequence of $k(n)$, then $g_{n}^{p(n)}\left(x_{2}\right) \in\left(x_{2}, c x_{1}\right)$ where $c=e(\varepsilon)$. This is impossible since $g_{n}^{p(n)}\left(x_{2}\right) \rightarrow x_{0}$.

We may assume that $g_{n}^{2 k(n)}$ satisfies (i) or (ii) in the definition of a convergence group. If $g_{n}^{2 k(n)} \rightarrow y_{0}$, so that $g_{n}^{-2 k(n)} \rightarrow y_{0}$ also, by Lemma 16, then $y_{0}=x_{1}$ or $y_{0}=x_{2}$ since $g_{n}^{2 k(n)}\left(x_{2}\right) \rightarrow x_{1}$. But then $g_{n}^{ \pm 2 k(n)}\left(x_{0}\right) \rightarrow y_{0}$ while the inequality $2 k(n) \leq p(n)$ implies that $g_{n}^{2 k(n)}\left(x_{0}\right) \in\left(x_{0}, c x_{2}\right)$ and $g_{n}^{-2 k(n)}\left(x_{0}\right) \in\left(\bar{c} x_{1}, x_{0}\right)$ for all large $n$. Hence at least one of $g_{n}^{2 k(n)}\left(x_{0}\right)$ and $g_{n}^{-2 k(n)}\left(x_{0}\right)$ does not tend to $y_{0}$. This is a contradiction, and it follows that $g_{n}^{2 k(n)} \rightarrow \tilde{g} \in G$, and $\tilde{g}\left(x_{2}\right)=x_{1}$.

We have $\tilde{g}\left(x_{0}\right)=x_{0}$ so that $\tilde{g}$ is parabolic. For if $\tilde{g}\left(x_{0}\right)=x_{3} \neq x_{0}$ then $g_{n}^{p(n)}\left(x_{3}\right) \rightarrow x_{0}$ and so $b_{n}=g_{n}^{p(n)+2 k(n)}\left(x_{0}\right) \rightarrow x_{0}$. But $b_{n}=g_{n}^{2 k(n)+p(n)}\left(x_{0}\right) \rightarrow$ $\tilde{g}\left(x_{2}\right)=x_{1} \neq x_{0}$, which gives a contradiction. Thus $\tilde{g}\left(x_{0}\right)=x_{0}$.

Applying the argument involving (8.1) to $x_{2}, x_{1}, x_{0}$, and $k(n)$ instead of $a, b, x_{0}$, and $p(n)$, and taking into account that this time $x_{0} \notin\left(x_{2}, x_{1}\right)$, we see that $g_{n}^{k(n)} \rightarrow \tilde{h} \in G$ and that $\tilde{h}^{2}=\tilde{g}$. Since $g$ and $\tilde{g}^{-1}$ are parabolic elements of $G$ fixing $x_{0}$ and taking $x_{1}$ onto $x_{2}$, it follows from Lemma 10 that $g=\tilde{g}^{-1}$, since the subgroup of elements of $G$ fixing $x_{0}$ can be conjugated to a Möbius group. Now $g_{n}^{-k(n)} \rightarrow h=\tilde{h}^{-1} \in G$, and $h^{2}=\tilde{g}^{-1}=g$. This proves Lemma 18.

8.5. To prove Lemma 14 when the $g_{n}$ are elliptic, choose positive integers $m(n)$ such that $0<m(n) \alpha(n)<1$ and $g_{n}^{m(n)}(1) \rightarrow-1$. This is possible since 
$\alpha(n)>0$ and $g_{n} \rightarrow \mathrm{Id}$. We may pass to a subsequence and assume that (i) or (ii) in the definition of a convergence group holds for $g_{n}^{m(n)}$. Suppose first that $g_{n}^{m(n)} \rightarrow h_{0} \in G$. By Lemma 18 and induction we conclude that there are functions $h_{n} \in G$ for $n \geq 1$ such that $h_{n}^{2}=h_{n-1}$. All the $h_{n}$ are parabolic, or all of them are elliptic.

8.5.1. If all the $h_{n}$ are parabolic with fixed point $z_{0}$, we find, by Lemma 10 , a homeomorphism $f$ of $\mathbf{T}$ onto $\overline{\mathbf{R}}$ with $f\left(z_{0}\right)=\infty$ such that all the elements of $G_{1}=f \circ G \circ f^{-1}$ fixing infinity are Möbius transformations. We may assume that $f \circ h_{0} \circ f^{-1}=T_{1}$, and then $f \circ h_{n} \circ f^{-1}=T_{2^{-n}}$ for all $n$. Thus $G_{1}$ contains $\left\{T_{s}: s=k / 2^{n}, k \in \mathbf{Z}, n \geq 0\right\}$. Since $G$ and hence $G_{1}$ is closed, we have $\mathscr{T} \subset G_{1}$, as required.

8.5.2. If all the $h_{n}$ are elliptic, we may assume that $h_{0}$ is conjugate to $R_{\beta}$ where $0<\beta<1$ and $\beta$ is rational. By Lemma 4, we may perform a preliminary conjugation of $G$ and assume that $h_{0}=R_{\beta}$. We set $S=\{e(\gamma): \gamma=$ $\left.k \beta / 2^{n}, 0 \leq \gamma<1, k \in \mathbf{Z}, n \geq 0\right\}$. For $z \in S, z=e\left(k \beta / 2^{n}\right)$, we define $f(z)=h_{n}^{k}(1)$. Since $h_{n+m}^{2^{m}}=h_{n}$, the functions $h_{n}$ commute, and so $f(z)$ does not depend on the representation of $z$ as an element of $S$.

Now one can verify that if $z_{1}, z_{2}, z_{3} \in S$ and $z_{2} \in\left(z_{1}, z_{3}\right)$, the $f\left(z_{2}\right) \in$ $\left(f\left(z_{1}\right), f\left(z_{3}\right)\right)$. Hence, if $f$ is continuous on $S$, we see as in the proof of Lemma 12 that $f$ is a homeomorphism of the dense subset $S$ of $\mathbf{T}$ onto some dense subset of $\mathbf{T}$, so that $f$ can be extended to a homeomorphism of $\mathbf{T}$ onto itself.

8.5.3. So we need to prove that $f\left(z_{n}\right) \rightarrow f(w)$ whenever $z_{n} \in S, w \in S$, and $z_{n} \rightarrow w$. If $z_{n}=e\left(k \beta / 2^{m}\right)$, where $k$ is odd, we set $u_{n}=h_{m}^{k}$. For simplicity, we may assume that $w=1$ and that the numbers $k / 2^{m}$ are positive and decrease to zero. Since $f(1)=1$, we need to show that $u_{n}(1) \rightarrow 1$. In any case, $u_{n+1}(1) \in\left(1, u_{n}(1)\right)$ so that $u_{n}(1) \rightarrow \alpha$, say, where $\alpha \in\left[1, u_{n}(1)\right)$ for all $n$.

Suppose that $\alpha \neq 1$. For any $n$, the functions $u_{1}, u_{2}, \ldots, u_{n}$ belong to a cyclic group generated by a function of finite order that is conjugate to a rotation. Hence $u_{n+1}(z) \in\left(u_{n+2}(z), u_{n}(z)\right)$ for all $n$ and all $z \in \mathbf{T}$. By passing to a subsequence we may thus assume that $u_{n} \rightarrow u$ uniformly on the circle where $u \in G$ and $u(1)=\alpha$. Since $u_{n+1}(z) \in\left(z, u_{n}(z)\right)$ for all large $n$ and all $z \in \mathbf{T}$, we have $u(z) \in\left[z, u_{n}(z)\right)$ for all large $n$ and all $z$. Since $u \neq$ Id, we have $u(z)=z$ for at most two values of $z$. It follows that for $n \geq j$, say, we have $u^{l}(z) \in\left[z, u_{n}^{l}(z)\right)$ if $1 \leq l \leq N=N(n)$. Here $N$ is the largest integer such that if $u_{n}$ is conjugate to $R_{\gamma}$ where $\gamma$ is rational and $0<\gamma<1$, then $N \gamma \leq 1$. Since $\gamma=k \beta / 2^{m} \rightarrow 0$, we have $N(n) \rightarrow \infty$, so that $u$ is of infinite order. As in the proof of Lemma 15, we deduce that $u$ is parabolic with fixed point $z_{0}$, say, where $1 \neq z_{0} \neq \alpha$. 
For any given $M=2^{l}>1$, there is $n \geq j$ such that $u_{n}^{M}(z) \in\left(z, u_{j}(z)\right)$ for all $z$ while $M<N(n)$. Hence $u^{M}(z) \in\left[z, u_{j}(z)\right]$ for all $z$ and all large $M$. We may find $z_{1}$ distinct from $z_{0}$ and choose $j$ so large that $z_{0} \notin\left[z_{1}, u_{j}\left(z_{1}\right)\right]$ since $u_{n}\left(z_{1}\right) \rightarrow u\left(z_{1}\right) \neq z_{0}$ as $n \rightarrow \infty$. Now we obtain a contradiction since $u^{M}\left(z_{1}\right) \rightarrow z_{0}$ as $M \rightarrow \infty$. Hence $f$ is continuous on $S$.

Furthermore, we find that $\left(f^{-1} \circ h_{n} \circ f\right)(z)=z e\left(\beta 2^{-n}\right)$, first for all $z \in S$ and hence for all $z \in \mathbf{T}$. It follows that $G_{1}=f^{-1} \circ G \circ f$ contains $\left\{R_{\theta}: \theta \in S\right\}$ and hence $\mathscr{R}$, since $G$ and thus also $G_{1}$ is closed.

8.5.4. Suppose next that $g_{n}^{m(n)} \rightarrow x_{0}$ and hence, by Lemma $16, g_{n}^{-m(n)} \rightarrow x_{0}$ on compact subsets of $\mathbf{T} \backslash\left\{x_{0}\right\}$, where $g_{n}^{m(n)}(1) \rightarrow-1$ as before. If $x_{0} \neq \pm 1$, we have a contradiction. Hence $x_{0}=1$ or $x_{0}=-1$. We set $z_{1}=-i$, $z_{2}=e(-1 / 8)$. Since $\alpha(n)>0$ and $0<m(n) \alpha(n)<1$, there are integers $k(n)$ with $0<k(n) \leq m(n)$ such that $g_{n}^{k(n)}\left(z_{1}\right) \rightarrow z_{2}$ as $n \rightarrow \infty$. Note that $g_{n}^{k(n)}\left(z_{1}\right) \in\left(z_{1}, g_{n}^{m(n)}\left(z_{1}\right)\right)$ and that $g_{n}^{m(n)}\left(z_{1}\right) \rightarrow x_{0}$. We pass to a subsequence and assume that $g_{n}^{k(n)} \rightarrow h \in G$ or that $g_{n}^{k(n)} \rightarrow x_{1}$ and $g_{n}^{-k(n)} \rightarrow x_{1}$. If $g_{n}^{k(n)} \rightarrow h$ then $h\left(z_{1}\right)=z_{2}$ and we may proceed in the same way as in the case when $g_{n}^{m(n)} \rightarrow h_{0} \in G$ and $h_{0}(1)=-1$ (see the beginning of subsection 8.5). So suppose that $g_{n}^{ \pm k(n)} \rightarrow x_{1}$. Since $g_{n}^{k(n)}\left(z_{1}\right) \rightarrow z_{2}$, we have $x_{1}=z_{1}$ or $x_{1}=z_{2}$. Hence $x_{1} \neq 1$, and we have $g_{n}^{k(n)}(1) \rightarrow x_{1}$ as $n \rightarrow \infty$.

We have $g_{n}^{k(n)}(1) \in(1,-1)$ since $0<k(n) \leq m(n)<1 / \alpha(n)$. Hence $x_{1} \in[1,-1]$. Since $z_{1}, z_{2} \notin[1,-1]$, we have a contradiction. It follows that $g_{n}^{k(n)}$ tends to a homeomorphism $h$ with $h\left(z_{1}\right)=z_{2}$.

This completes the proof of Lemma 14. Hence we have proved Theorem 1 for nondiscrete groups.

8.6. Suppose that $G$ is an uncountable convergence group on $\mathbf{T}$. Then we can show as in $[3, \S 11]$ that $G$ is nondiscrete. The normal family argument for a sequence $h_{n} \in G$ used there is replaced by the use of the definition of a convergence group, and the same conclusion follows. Hence $(v)$ of Theorem 1 now follows from (iv) of Theorem 1.

8.7. If $G$ is a closed noncyclic abelian convergence group, we may proceed as in $[3, \S 8]$. Instead of [3, Lemma 9], we use Lemma 14 with all the $g_{n}$ elliptic. The proof in [3] goes through without any other essential changes and shows that $G$ is conjugate to a Möbius group. This together with Lemmas 4 and 5 proves case (ii) of Theorem 1.

For completeness, we shall outline the proof. Let fix $(g)$ denote the set of fixed points of $g$. If $g, h \in G$, so that $g \circ h=h \circ g$, it is seen that

$$
h(\operatorname{fix}(g))=\operatorname{fix}(g) \text {. }
$$

If some $g \in G$ has exactly one fixed point, then all the elements of $G$ fix that point. Then $G$ is topologically conjugate to a Möbius group by case (i) of 
Theorem 1, which we proved already. Otherwise, by Lemma 1, exactly one of the following three possibilities arises:

Case I. There is $g \in G$ with exactly two fixed points such that $g^{2} \neq \mathrm{Id}$;

Case II. There is no $g$ as in Case I but there is $g \in G$ with exactly two fixed points such that $g^{2}=\mathrm{Id}$; or

Case III. Any $g \in G \backslash\{$ Id $\}$ has no fixed points.

In Cases I and II we may assume that $G$ is defined on $\overline{\mathbf{R}}$ and that $g$ fixes 0 and $\infty$. By case (i) of Theorem 1, we may perform a preliminary conjugation and assume that the subgroup $G_{1}=\{h \in G: h(\infty)=\infty\}$ of $G$ consists of linear functions. Furthermore, since $h(\{0, \infty\})=\{0, \infty\}=$ fix $(g)$ for all $h \in G$, we see that any element of $G_{1}$ fixes both 0 and $\infty$ and is therefore equal to $M_{a}$ for some real nonzero $a$, while any element of $G \backslash G_{1}$ interchanges 0 and $\infty$.

In Case I, at least one of $g, g^{2}$, and $g^{-2}$ is of the form $M_{b}$ for some $b>1$, so that $h\left(b^{n} x\right)=b^{n} h(x)$ whenever $h \in G \backslash G_{1}, x \in \mathbf{R}$, and $n$ is an integer. Since $h(\infty)=0$, we have $h\left(b^{n}\right) \rightarrow 0$ as $n \rightarrow \infty$ while $\left|b^{n} h(1)\right| \rightarrow \infty$. This contradiction shows that $G=G_{1}$, which is a Möbius group.

In Case II, we may assume that $G \neq G_{1}$. Then $G_{1}$ cannot contain a function $M_{b}$ for $b>1$, for that would lead to a contradiction as above. Thus $G_{1}$ can only contain Id and $M_{-1}$. If $G_{1}=\{\mathrm{Id}\}$ then $G$ is cyclic of order two, which contradicts our assumption that $G$ is not cyclic. Thus $G=\left\{\right.$ Id $, M_{-1}, g_{1}$, $\left.g_{1} \circ M_{-1}\right\}$ where $g_{1} \in G \backslash G_{1}$ and $g_{1}^{2}=$ Id. Thus $G$ has the algebraic structure of the Vierergruppe. Recall that $g_{1}$ and $g_{1} \circ M_{-1}$ interchange 0 and $\infty$. We may assume that $g_{1}$ is sense-reversing since one of $g_{1}$ and $g_{1} \circ M_{-1}$ is.

By Theorem 1 for cyclic groups, we may conjugate $G$ to the group $G^{\prime}=$ $\{$ Id $, h, H, h \circ H\}$ where $H(x)=1 / x$ is the conjugate of $g_{1}$ while $h$, the conjugate of $M_{-1}$, is a strictly decreasing function fixing 0 and $\infty$. We have $h^{2}=$ Id and $h \circ H=H \circ h$.

We set $f(x)=x$ for $x \geq 0$ and $f(x)=-h(x)$ for $x<0$. Then $f(1 / x)=$ $1 / f(x)$ and $f \circ h \circ f^{-1}=M_{-1}$. Thus $f \circ G^{\prime} \circ f^{-1}$ is a Möbius group.

In Case III, it suffices to show that $G$ is finite or nondiscrete, thus reducing the problem to Theorem 2 or to case (iv) of Theorem 1, both of which have been proved already. We assume that $G$ is infinite and show that $G$ is nondiscrete.

In any case, Theorem 1 for cyclic groups shows that each $g \in G \backslash\{$ Id $\}$ is topologically conjugate to a rotation $R_{\alpha}$ where $0<\alpha<1$. If some such $\alpha$ is irrational, then clearly $G$ is nondiscrete. Suppose therefore that all such numbers $\alpha$ are rational. Then all the elements of $G$ have finite order. Now the proof of [3, Lemma 7] shows that since $G$ is not cyclic, it follows from topological reasons that $G$ contains no element of maximal order. Thus $G$ contains a sequence $g_{n}$ such that $g_{n}$ is conjugate to $R_{1 / N(n)}$ where $N(n)$ strictly increases to infinity as $n \rightarrow \infty$.

We claim that $g_{n} \rightarrow$ Id uniformly on $\mathbf{T}$ as $n \rightarrow \infty$, and this then shows that $G$ is nondiscrete. Recall the notation (2.1). As in the proof of [3, Lemma 
8], we see that for topological reasons

$$
g_{m+1}(z) \in\left(z, g_{m}(z)\right) \text { for all } z \in \mathbf{T} .
$$

Now we follow closely the argument given in [3, subsection 8.6]. If $N(m) \geq$ 6 , set $z_{1}=1, z_{2}=g_{m}^{2}(1)$, and $z_{3}=g_{m}^{4}(1)$, so that the $\operatorname{arcs}\left(z_{j}, g_{m}\left(z_{j}\right)\right)$ have disjoint closures for $1 \leq j \leq 3$. If $n>m$, then

$$
g_{n}\left(z_{j}\right) \in\left(z_{j}, g_{m}\left(z_{j}\right)\right) \text { for } 1 \leq j \leq 3 .
$$

By the definition of a convergence group, we may pass to a subsequence and assume that $g_{n} \rightarrow g$ uniformly on $\mathbf{T}$ as $n \rightarrow \infty$, where the homeomorphism $g$ is an element of the closed group $G$. Note that the condition (8.2), where $m$ and the $z_{j}$ are fixed, excludes the possibility that $g_{n} \rightarrow x_{0}$ locally uniformly in $\mathbf{T} \backslash\left\{y_{0}\right\}$ for some $x_{0}, y_{0} \in \mathbf{T}$.

If $g \neq$ Id then $|g(z)-z| \geq \delta>0$ for all $z \in \mathbf{T}$, since $g$ has no fixed points by our assumption in Case III. There is $n_{0}$ such that if $n \geq n_{0}$, then $\left|g_{n}(z)-g(z)\right|<\delta / 2$ and thus $\left|g_{n}(z)-z\right|>\delta / 2$ for all $z \in \mathbf{T}$. We can write $g_{n}=h_{n} \circ R_{1 / N(n)} \circ h_{n}^{-1}$, where $h_{n}$ is a homeomorphism of $\mathbf{T}$, and this gives

$$
\left|h_{n}\left(d_{n} z\right)-h_{n}(z)\right| \geq \delta / 2>0 \text { for all } z \in \mathbf{T} \text {, for } n \geq n_{0},
$$

where $d_{n}=e(1 / N(n))$. Now we get a contradiction by choosing $n$ so large that $N(n) \delta / 2>2 \pi$ and applying (8.3) to $z=e(k / N(n))$ for $0 \leq k \leq N(n)-1$. This proves that $g=I d$.

Thus $g_{n} \rightarrow$ Id and $G$ is nondiscrete, as asserted. This completes the proof of Theorem 1 for closed noncyclic abelian groups, and as remarked at the beginning of subsection 8.7, case (ii) of Theorem 1 follows.

The proof of Theorem 1 is thus complete.

\section{REFERENCES}

1. F. W. Gehring and G. J. Martin, Discrete quasiconformal groups. I, Proc. London Math. Soc. (3) 55 (1987), 331-358.

2. A. Hinkkanen, Uniformly quasisymmetric groups, Proc. London Math. Soc. (3) 51 (1985), 318-338.

3. - The structure of certain quasisymmetric groups, Mem. Amer. Math. Soc. (to appear).

4. G. J. Martin and P. Tukia, Convergence and Möbius groups, Holomorphic Functions and Moduli II, MSRI publ. 11, Springer, New York, 1988, pp. 113-140.

5. P. Tukia, Homeomorphic conjugates of Fuchsian groups, J. Reine Angew. Math. 391 (1988), $1-54$. 